\title{
Flow characteristics and scaling past highly porous wall-mounted
}

\section{fences}

\author{
Eduardo Rodríguez-López *1 \\ Paul J.K. Bruce ${ }^{1}$ \\ Oliver R.H. Buxton ${ }^{1}$
}

${ }_{4} \quad$ *: Corresponding author: eduardo.rodriguez-lopez12@imperial.ac.uk

${ }_{5}{ }^{1}$ : Department of Aeronautics. Imperial College London. Exhibition Road, London SW7 2 AZ. United Kingdom.

\section{Abstract}

8 An extensive characterization of the flow past wall-mounted highly porous fences based on single- and multi-scale 9 geometries has been performed using hot-wire anemometry in a low-speed wind tunnel. Whilst drag properties 10 (estimated from the momentum equation) seem to be mostly dependent on the grids' blockage ratio; wakes of 11 different size and orientation bars seem to generate distinct behaviours regarding turbulence properties. Far 12 from the near-grid region, the flow is dominated by the presence of two well-differentiated layers: one close to 13 the wall dominated by the near-wall behaviour and another one corresponding to the grid's wake and shear ${ }_{14}$ layer, originating from between this and the freestream. It is proposed that the effective thickness of the wall 15 layer can be inferred from the wall-normal profile of root-mean-square streamwise velocity or, alternatively, from the wall-normal profile of streamwise velocity correlation. Using these definitions of wall-layer thickness enables us to collapse different trends of the turbulence behaviour inside this layer. In particular, the root-mean-square level of the wall shear stress fluctuations, longitudinal integral length scale and spanwise turbulent structure are shown to display a satisfactory scaling with this thickness rather than with the whole thickness of the grid's wake. Moreover, it is shown that certain grids destroy the spanwise arrangement of large turbulence structures in the logarithmic region which are then re-formed after a particular streamwise extent. It is finally shown that for fences subject to a boundary layer of thickness comparable to their height the effective thickness of the wall layer scales with the incoming boundary layer thickness. Analogously, it is hypothesized that the growth rate of the internal layer is also partly dependent on the incoming boundary layer thickness. 


\section{Introduction}

2 Wind-tunnel testing usually requires the generation of artificially thick turbulent boundary layers (TBL). This 3 is traditionally obtained by means of obstacles in such a way that their drag removes momentum from the fluid 4 thickening the TBL (e.g. Counihan, 1969). Alternatively one can generate that loss of momentum by integrating 5 an increased level of skin friction by means of roughness over a certain streamwise extent (e.g. Jimenez, 2004) or 6 simulate it with the inclusion of freestream turbulence (Dogan et al., 2016). Whilst the two latter methods may present certain advantages, they require a longer streamwise extent and a more complex set-up than the former. Apart from the pioneering work of Counihan (1969), several other examples can be found in the literature considering thickening of TBL by immersion of obstacles (for a review see Hunt and Fernholz, 1975).

In a recent work, Rodríguez-López et al. (2016a,b) showed that, in these cases, the way obstacles' wakes interact with the near-wall region may have important implications in the length of the adaptation region. This region is understood as the streamwise fetch required for the obstacle's influence to be "forgotten" and 3 canonical TBL properties recovered. Whilst this state is relatively easy to be achieved in short wind tunnels if small trips are used (Erm and Joubert, 1991; Schlatter and Örlü, 2012); it is increasingly difficult with larger trips. Hence the possibility of reducing the adaptation region's length is of interest. Rodríguez-López et al. (2016a,b) associated shorter adaptation regions with the lack of bulk recirculating fluid downstream of the obstacles. Furthermore, they showed that these situations are more likely to appear in cases where the wake of the obstacles is prevented from strongly influencing the near-wall region. This interaction is prevented for low-blockage (approximately 30\%) obstacles in which the fluid bled through the obstacle makes it possible to avoid wall-normal interaction of wake and near-wall regions.

A possible way of further studying this problem is the generation of different degrees of interaction between the obstacle's wake and the wall whilst simultaneously avoiding bulk recirculation. This opens the possibility of using wall-mounted porous fences. These kinds of obstacles have been extensively used as a passive way of controlling various environmental and civil flows (e.g. Ranga-Raju et al., 1988; Li and Sherman, 2015). Downstream of turbulence-generating grids ${ }^{1}$ the presence of strong recirculation is controlled by their blockage ratio $\sigma$ ( $\sigma$ is taken as the relationship between the solid area of the fence and the area of the smallest frame that can surround it). Castro (1971) offered experimental evidence showing that no recirculation appeared for $\sigma \lesssim 70 \%$. The drag coefficient of these grids, which, as mentioned above has implications for their ability as boundary layer thickening elements, also presents a maximum at this blockage ratio.

Maximizing the drag coefficient is equivalent to maximizing the sheltering properties of the grid close downstream of them. This is usually one of the main objectives of their engineering applications. Consequently, the flow past porous fences has probably been a victim of its own engineering success and a large number of studies has been focused on grid's blockage of about 60 or 70\% (Seginer, 1972; Ranga-Raju et al., 1988; Keylock et al., 2012; Li and Sherman, 2015, amongst others). However, little attention has been paid to lower porosity fences. Analogously, most of the studies are concentrated in a region close downstream of the grids, whereas the far

\footnotetext{
${ }^{1}$ For readability, the terms grid or porous fence will be used equivalently throughout this paper
} 
field development remains an open question. Curiously, the main interest for TBL thickness control is focused on the far-field development of low-porosity fences.

Following the aforementioned recommendations, it seems reasonable to fix the grids' blockage at $30 \%$ with the double objective of (i) avoiding fluid recirculation and (ii) preventing wake/near-wall interaction hence minimizing the extent of the adaptation region. However, fixing blockage ratio is far from finalising the grids design, particularly if one considers the turbulent properties downstream of the fences. The way the porosity is distributed along the grids can significantly influence the turbulent properties in their wakes and therefore the way they interact with the near-wall region. A few studies have previously proposed the use of non-uniform profiles of blockage (Wilson, 1987) as well as multiscale based approaches (Keylock et al., 2012).

It is precisely from this perspective that new designs can be studied. During the last decade, several researchers have proposed novel practical applications to fractal-based multiscale geometries (first explored by Hurst and Vassilicos, 2007). Some of these applications include: noise of aerodynamic spoilers (Nedić et al., 2012), study of wakes (Dairay et al., 2015), aerodynamic performance (Nedić and Vassilicos, 2015), stirred tanks (Steiros et al., 2017) or vortex shedding study (Melina et al., 2016). Furthermore, they contributed to the discovery of new scaling laws for the location and intensity of the turbulence intensity peak downstream of grids (Mazellier and Vassilicos, 2010; Gomes-Fernandes et al., 2012).

From these examples, one can assume that multiscale-based geometries present a clear potential to modify turbulent properties (intensity, spectra, length scales, etc.) for a fixed blockage ratio (which is essential to achieve a fair comparison in terms of flow past porous fences). Thus, the present paper converges these three methodologies. Firstly, studies of the evolution of tripping conditions necessitate the generation of large wakes which may experience different degrees of interaction with the near wall region. Secondly, highly porous fences may result in satisfactory devices for that purpose once their blockage ratio is matched in order to ensure a fair comparison. Thirdly, a possible way of modifying turbulent properties for a fixed blockage ratio resides on the use of multiscale geometries which have been successfully applied before for a certain number of applications.

The main aim of this study is twofold: firstly, to assess the validity of various highly porous fences as devices to generate a thicker TBL. This part is based on the drag characterization of the grids, which is directly related with the modification of the TBL momentum thickness far downstream. Furthermore, links are proposed between this and a previous study (Rodríguez-López et al., 2017) which considered the evolution of the wall shear stress properties downstream of the same geometries. Secondly, a comprehensive flow characterization is conducted paying special attention to the turbulence properties and scaling of the adaptation region. Along these lines, one- and two-points simultaneous hot-wire anemometry are used to provide answers about the scaling magnitudes of the spatially evolving TBL downstream of highly porous fences. 


\section{Experimental set-up}

\subsection{The wind tunnel}

3 The experiments are conducted at Imperial College London in a wind tunnel of $0.91 \times 0.91 \mathrm{~m}^{2}$ section and $4.8 \mathrm{~m}$

4 length, with freestream velocity $U_{\infty} \approx 10 \mathrm{~m} \mathrm{~s}^{-1}$. At these conditions, the incoming freestream turbulence level

5 is $\leq 0.05 \%$. The same wind tunnel has been broadly documented before (e.g. Mazellier and Vassilicos, 2010;

6 Valente and Vassilicos, 2014; Rodríguez-López et al., 2016b).

A wooden flat plate of thickness $16 \mathrm{~mm}$ is mounted vertically spanning the whole wind tunnel with an elliptic $8 \quad(10: 1)$ leading edge to avoid separation, and a trailing edge flap in order to modify the position of the stagnation 9 point on the leading edge and control the pressure gradient along the plate. A strip of sand paper $20 \mathrm{~mm}$ long

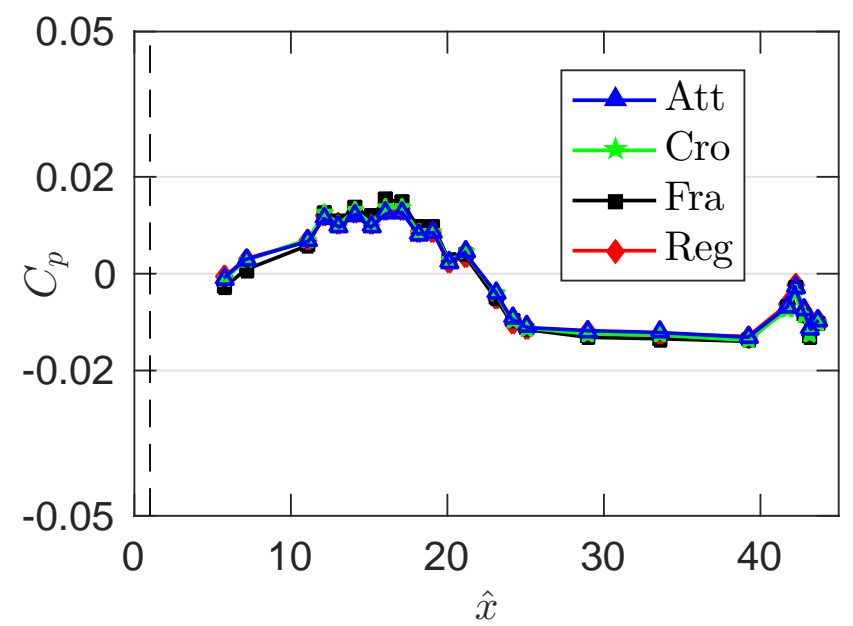

(a)

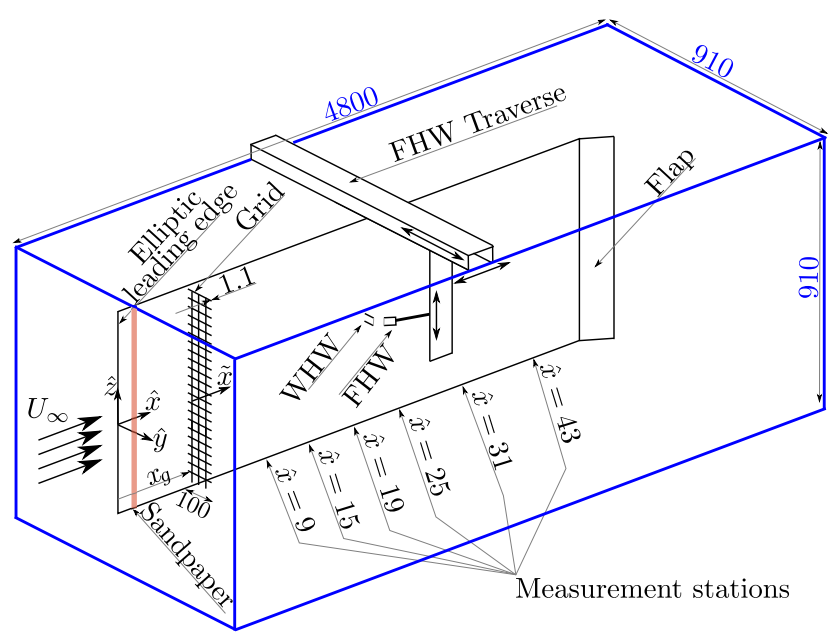

(b)

Figure 1: Left column: (a) Pressure distribution along the flat plate for different configurations with or without grid (c.f. section 2.2 for a description of the grids). The dashed black line shows the location at which the grids were installed. (b) Sketch of the wind-tunnel arrangement (Not to scale, all dimensions in mm), flow goes left to right. $\hat{x}=x / h$ is defined as the streamwise distance from the leading edge normalized with the grid height and $\tilde{x}=\left(x-x_{g}\right) / h$ denotes the streamwise distance from the grid normalized with its height $h=100 \mathrm{~mm}$. WHW symbolizes the wall hot wire and FHW the free hot wire (c.f. section 2.3 for details).

For the first part of the study, the different grids are all located at $x_{g}=100 \mathrm{~mm}$ where the thickness of the boundary layer is negligible with respect to the height of the grid. In order to study the influence of the incoming boundary layer thickness, the grids can also be mounted at different $x_{g}$ positions where the boundary 
1 layer has thickness $\delta^{I}$ and momentum thickness $\theta^{I}$. et al. (2017).

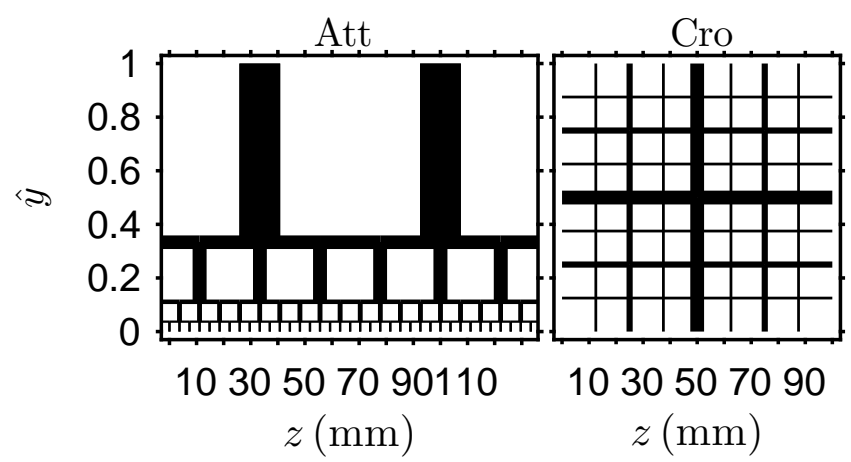
from the grid normalized with its height.

\subsection{The grids}

The coordinate system defines $x$ as the streamwise distance downstream of the plate leading edge, $y$ as the wall-normal distance from the plate, and $z$ as the spanwise distance relative to the centreline. Mean quantities are denoted by a horizontal bar while the fluctuating part is shown with a superscript $/\left(\right.$ e.g. $\left.a(t)=\bar{a}+a^{\prime}(t)\right)$. The subscript rms is used to express the root-mean-square level. The mean wall shear stress is expressed by $\bar{\tau}_{w}$ and the friction velocity $u_{\tau}=\sqrt{\bar{\tau}_{w} / \rho}$ where $\rho \approx 1.17 \mathrm{kgm}^{-3}$ is the fluid viscosity. The superscript + is used for magnitudes expressed in wall units, i.e. non-dimensionalized with the kinematic viscosity, $\nu \approx 1.5 \times 10^{-5} \mathrm{~m}^{2} \mathrm{~s}^{-1}$, and the friction velocity, $u_{\tau}$. In particular a wall unit is defined as $\delta_{\nu}=\nu / u_{\tau}$, thus $y^{+}=y / \delta_{\nu}, u^{+}=u / u_{\tau}$ and the time, $t^{+}=t u_{\tau}^{2} / \nu$. Throughout the paper quantities normalized with the grid height, $h$, and/or the freestream velocity are designed by the superscript $\wedge$, e.g. $\hat{u}=u / U_{\infty}$, or $\hat{x}=x / h$, which is defined as the streamwise distance from the leading edge normalized with the grid height. $\tilde{x}=\left(x-x_{g}\right) / h$ denotes the streamwise distance

Four different porous fences are designed and mounted. The grids are made of two pieces of $400 \mathrm{~mm}$ span and with a height $h=100 \mathrm{~mm}$ mounted together at each side of the centreline up to a final span of $800 \mathrm{~mm}(89 \%$ of the tunnel span). Special attention is paid to ensure the joint between the two pieces is as solid as possible. The total spanwise extent is obtained by the periodic repetition of the basic patterns described below; all the grids have the same blockage ratio $\sigma=30 \%$. The grids could be mounted normal to the flat plate at several streamwise locations in the range $0.1 \leq x_{g} \leq 3.3 \mathrm{~m}$ by means of thin legs (threaded rods of $3 \mathrm{~mm}$ diameter) which passed to the rear side of the plate where they were held by a nut. Figure 2 shows the grids' basic patterns and Table 1 summarizes their main geometrical parameters. The same grids were also used by Rodríguez-López

Figure 2: Drawings of the basic pattern of the four tested grids. Note that thickness and length of the bars is to scale within the pictures. $z=0$ corresponds to the wind-tunnel mid plane.

Att. This grid is obtained based on the attached eddy hypothesis of Townsend (1976) stating that eddy size would scale with its distance from the wall. The grid is designed by stacking four squares with lengths $l_{i}$ $(i=0,1,2,3)$, where $l_{i}$ equals the distance from their centre to the wall. This produces a geometric distribution 


\begin{tabular}{|r|r|c|c|c|c|}
\cline { 3 - 6 } \multicolumn{2}{c|}{} & \multicolumn{5}{c|}{ Grids } \\
\hline \hline Description & Symbol & Att & Cro & Fra & Reg \\
\hline Height & $h(\mathrm{~mm})$ & 100 & 100 & 100 & 100 \\
\hline Blockage ratio & $\sigma(\%)$ & 30 & 30 & 30 & 30 \\
\hline Sumber of iterations & $N$ & 4 & 3 & 3 & 1 \\
\hline Spanwise periodicity & $\Delta z(\mathrm{~mm})$ & 66.6 & 100 & 100 & 33.3 \\
\hline \multirow{3}{*}{ Thickness of bars } & $t_{0}(\mathrm{~mm})$ & 14.8 & 5.6 & 5 & 5 \\
& $t_{1}(\mathrm{~mm})$ & 4.93 & 2.50 & 2.24 & - \\
& $t_{2}(\mathrm{~mm})$ & 1.64 & 1.12 & 1 & - \\
& $t_{3}(\mathrm{~mm})$ & 0.82 & - & - & - \\
\hline & $t_{0}(\mathrm{~mm})$ & 66.7 & 50 & 57 & 33.3 \\
& $t_{1}(\mathrm{~mm})$ & 22.2 & 25 & 28.5 & - \\
& $t_{2}(\mathrm{~mm})$ & 7.4 & 12.5 & 28.5 & - \\
& $t_{3}(\mathrm{~mm})$ & 3.7 & - & - & - \\
\hline
\end{tabular}

Table 1: Summary of geometrical parameters for the various grids.

1 design in order to avoid a large blockage in the outer region of the flow which would be significantly at odds 2 with the other designs and not representative of a TBL. In order to appropriately scale the bar thickness, $t_{i}$ this 3 is kept as a constant fraction $\left(l_{i} / t_{i}=4.51\right)$ of the squares' length throughout the four iterations. Note that this 4 by keeping $l_{i} / t_{i}$ constant, the grid does not match Townsend's eddy hierarchy in the outer part of the boundary 5 layer.

Cro. The grid design is based on a fractal cross pattern first described by Hurst and Vassilicos (2007). 7 The only free parameters to fix are the number of iterations (which is kept to 3 due to the impossibility of 8 manufacturing bars of smaller thickness); the thickness of the largest bar $t_{0}=5.6 \mathrm{~mm}$ and the thickness ratio $9 t_{r}=t_{0} / t_{2}=5$ (relation of the largest and smallest thickness). This grid has also been employed as a turbulence generator for combustion of opposing jets Goh et al. (2013, 2014).

Fra. The grid design is based on the fractal square pattern described by Hurst and Vassilicos (2007). The number of iterations, 3 , and thickness ratio $\left(t_{r}=5\right)$ are chosen to be the same as the Cro case and the thickness of the largest bar as similar as possible $\left(t_{0}=5 \mathrm{~mm}\right)$. This parameter cannot be varied independently from the blockage ratio which has to be $\sigma=30 \%$ for every grid. Turbulent properties of this grid have been extensively studied in the past years (e.g Hurst and Vassilicos, 2007; Valente and Vassilicos, 2014; Melina et al., 2016, amongst others).

Reg. In order to characterize the effect of different bars arrangements; a regular grid is designed matching the same blockage $(\sigma=30 \%)$ and thickness $(t=5 \mathrm{~mm})$ of the multiscale cases.

\subsection{Hot wire anemometry}

Two single-component hot-wire anemometers operated in constant temperature mode are employed to perform velocity measurements.

A first hot wire with diameter $d_{w}=5 \mu \mathrm{m}$ and length $l_{w} \approx 1 \mathrm{~mm}$ is soldered onto the prongs of a 55P05 boundary layer probe from Dantec Dynamics. This sensor is mounted on a linear traverse enabling its positioning at every point of the wind tunnel in the $y$ and $z$ directions and $9 \leq \hat{x} \leq 43$ in the streamwise direction. This 
traverse system is composed of three endless screws moved by steppermotors and controlled by a computer. The system enables the hot wire to be positioned and moved automatically during the experiment with a smallest displacement better than $2 \mu \mathrm{m}$ for every direction. The sensor is placed in the near-wall vicinity by means of a microscope but the accurate wall-probe relative position is extracted from the velocity profile following Rodríguez-López et al. (2015). Using an overheat ratio OHR=1.8 and sampling at $100 \mathrm{kHz}$ for $60 \mathrm{~s}$ and low-pass filtering at $f_{f}=30 \mathrm{kHz}$ enables resolving the smallest scales of the fluid estimated by $0.2<t^{+}=u_{\tau}^{2} /\left(\nu f_{f}\right)<0.4$ for the different configurations and streamwise locations. Note that, in this flow configuration, the kolmogorov and inner scaling may be decoupled since the grid acts as a turbulence generator, not scaling in inner units. This could be a limitation in order to assess the hot-wires resolution as a function of $l^{+}$for regions of the flow immersed in the grids' wakes. Nevertheless, apart from the wall-shear stress measurements (which will be discussed below), the manuscript will only consider large-scale quantities such as the integral length scale or two-point correlations, which are substantially less affected by the limited resolution of hot wires. This hot wire is calibrated statically against a straight Pitot tube located in the freestream. This sensor will be called FHW (free hot wire) for brevity.

A second sensor is built by attaching the body of a 55P01 Dantec Dynamics hot wire to a linear traverse mounted on the back of the flat plate. The prongs are passed through two holes and a thin coat of wax is applied to avoid any flow leakage. The sensor is made from a $d_{w}=5 \mu \mathrm{m}$ Wollaston wire in-house etched to a length of $l_{w}=0.82 \pm 0.05 \mathrm{~mm}$. The value of $l_{w} / d_{w} \approx 164$ is lower than the recommended value of 200 (Brunn, 1995). A correction to this effect could be applied based on the scheme proposed by Hultmark et al. (2011) and Miller et al. (2014). However, this correction only modifies the measured values by a small amount (e.g. $0.7 \%$ in the $u_{r m s}^{\prime}$ value) hence it can be avoided since it is not the largest contribution to the error of this sensor. Furthermore this correction scheme cannot correct the instantaneous values of the fluctuations used for two-point measurements. The linear traverse enables positioning of the hot wire in the range $0.18 \leq y \leq 3 \mathrm{~mm}$ with $10 \mu \mathrm{m}$ precision. The same values of OHR and $f_{f}$ are kept as in the FHW case. However, calibration of this device is significantly more challenging; the sensor is calibrated against the quasi-laminar boundary layer developing over the surface of a purpose-built small flat plate. Details of the calibration technique are given in the appendix A. The calibration was repeted before or after every experiment ensuring that the temperature drift between experiment and calibration was smaller than $0.5 \mathrm{~K}$. This sensor will be referred to as WHW (wall hot wire) for brevity.

The set-up enables us to position both hot wires in the wall vicinity (and close to each other) such that simultaneous sampling of them is possible. This enables the measurement of two-point statistics both in the wall-normal and the spanwise directions by moving the FHW and leaving the WHW at a fixed position. To this effect, the inner hot wire is placed at a few wall untis from the wall and the FHW is traversed in the $y$ or $z$ directions for wall-normal or spanwise correlation respectively by means of the traverse system described above. Additionally, the independent traverse system of the WHW enables its positioning at various wall-normal locations. The points affected by the interaction of both sensors are discarded.

Direct measurements of the mean wall shear stress $\bar{\tau}_{w}$, are also available from a previous study (Rodríguez- 
6 is available.

\begin{tabular}{|r|c|c|c|c|c|c|c|}
\hline Grid & $\hat{x}=9$ & $\hat{x}=15$ & $\hat{x}=19$ & $\hat{x}=25$ & $\hat{x}=31$ & $\hat{x}=36$ & $\hat{x}=43$ \\
\hline Att & 0.3299 & 0.3035 & 0.3065 & 0.3151 & 0.3163 & 0.3301 & 0.3504 \\
\hline Cro & 0.3480 & 0.3251 & 0.3253 & 0.3286 & 0.3259 & 0.3491 & 0.3557 \\
\hline Fra & 0.3173 & 0.3068 & 0.3136 & 0.3167 & 0.3136 & 0.3100 & 0.3141 \\
\hline Reg & 0.3341 & 0.3091 & 0.3119 & 0.3132 & 0.3042 & 0.3066 & 0.3145 \\
\hline
\end{tabular}

Table 2: Values of the friction velocity $u_{\tau}$ for various grids and streamwise locations extracted from RodríguezLópez et al. (2017).

\title{
3 Results
}

8 This section will be divided as follows: in the first place, the drag of the various porous fences will be studied in 9 section 3.1. The turbulence properties downstream of different grids will be presented in section 3.2. The two

López et al., 2017) with an approximate accuracy of $4 \%$ on the determination of $u_{\tau}$. For completness, these values are summarized in Table 2 for the various grids and streamwise locations. These values are better than extrapolation from the mean velocity profile which may be ill-posed in strongly disrupted cases downstream of the grids. However, Rodríguez-López et al. (2017) show that estimations of $u_{\tau}$ from the velocity profile using the method proposed by Rodríguez-López et al. (2015) can give reasonable estimations of $u_{\tau}$ if no other method

\begin{abstract}
layers hypothesized in section 1 are characterized in section 3.3. Later on, the validity of this scaling is tested for the fluctuations of the wall shear stress (section 3.4), the longitudinal integral length scale (section 3.5) and the spanwise turbulent structure (section 3.6). Finally, the influence of the incoming boundary layer will be studied in section 3.7 .
\end{abstract}

\subsection{Drag of grids}

The ultimate goal of any device to thicken TBL is to precisely increase its Reynolds number for fixed freestream velocity, and streamwise distance. A simple analysis of the integral momentum equation for a naturally growing zero pressure gradient boundary layer shows that the momentum thickness $\theta$, (and analogously the Reynolds number $\left.R e_{\theta}=\theta U_{\infty} / \nu\right)$ increases as a consequence of the momentum lost by friction with the wall. The inclusion of obstacles (such as grids or trips) introduces an additional loss of momentum equal to their drag which contributes to increasing $R e_{\theta}$. Consequently, there is a direct link between the drag of a certain grid and its applicability as a device to increase TBL thickness. From another perspective, a common way to assess the performance of wall-mounted fences is to characterize their drag $D$ per unit of spanwise length. This provides information about how much momentum is removed from the fluid and therefore it can give an estimation of the sheltering properties of a given fence. Hence a simple characterization in terms of drag coefficient may provide significant answers in terms of the fence performance, both as boundary layer modifier (in the far field) and also as sheltering fence (in the near field). Here, sheltering is understood as the capacity of the grids to reduce the flow velocity in their vicinity in order to, for instance, decrease the erosion generated by the wind (Li and 
Sherman, 2015).

Let us first define the drag coefficient per unit length as $C_{d}=2 D /\left(\rho U_{\infty}^{2} h\right)$. Despite some studies (e.g. Good and Joubert, 1968; Ranga-Raju et al., 1988) proposing the use of the local friction velocity $u_{\tau}$ for the non-dimensionalization of $D$ for various degrees of immersion; the election of $U_{\infty}$ seems more adequate for low values of $\delta^{I} / h$ such as those tested in this study. Furthermore, it also facilitates the comparison with other researchers given the difficulties of finding $u_{\tau}$ for certain experimental and atmospheric situations.

Contrary to studies in which the drag could be measured directly (Guan et al., 2003), the drag in this paper will be estimated from the momentum equation. This methodology has been previously followed by several researchers (see, e.g. Woodruff et al., 1963; Seginer, 1972; Dong et al., 2008) under different assumptions and/or experimental limitations. The procedure considers the integral streamwise momentum equation over a domain extending from $\tilde{x}_{1}=-1$ to $\tilde{x}_{2}=8$ in the streamwise direction and from $y=0$ to $y \rightarrow \infty$ in the wall-normal direction. Two hypotheses are required: (i) neglect the contribution of the pressure based on the $C_{p}$ evolution shown in figure 1(a). (ii) Model the contribution of the wall shear stress proportional to the wall shear stress that would appear in a naturally growing boundary layer over the same streamwise extent $\left(\bar{\tau}_{w}(x) \propto \bar{\tau}_{w}^{N}(x)\right)$. There, the constant of proportionality being is relationship between $\bar{\tau}_{w}$ at the outlet of the domain in the cases with and without grid: $\tau^{G N}=\bar{\tau}_{w}\left(x_{2}\right) / \bar{\tau}_{w}^{N}\left(x_{2}\right)$. Furthermore, we define the momentum thickness $\theta=\int_{0}^{\infty}[\hat{u}(1-\hat{u})] \mathrm{d} y$. Under these two hypotheses, we can calculate the drag coefficient as

$$
C_{d} \approx \frac{2}{h}\left[\theta_{2}-\theta_{2}^{N} \tau^{G N}\right]
$$

where the superindex $N$ symbolizes the naturally growing ZPG TBL over the same domain. Complete details of the procedure and discussion on the hypotheses can be found in the apendix B.

\begin{tabular}{|r|c|c|c|c|}
\hline & Att & Cro & Fra & Reg \\
\hline$\theta_{2}(\mathrm{~mm})$ & 23.4 & 21.2 & 24.2 & 24.2 \\
\hline$\theta_{D}(\mathrm{~mm})$ & 21.5 & 19.0 & 22.4 & 22.2 \\
\hline$\left(\theta_{2}-\theta_{D}\right) / \theta_{2}(\%)$ & 8.21 & 10.1 & 7.33 & 8.15 \\
\hline$C_{d}$ & 0.43 & 0.38 & 0.45 & 0.44 \\
\hline
\end{tabular}

Table 3: Values of momentum thickness $\theta_{2}$, at $x_{2}$ (outlet of the domain), $\theta_{D}=\left[\theta_{2}-\theta_{2}^{N} \tau^{G N}\right]=h C_{d} / 2$ and drag coefficient $C_{d}$ for the various grids. The grids are always located at $x_{g}=100 \mathrm{~mm}$ where the incoming boundary layer thickness $\delta^{I} \approx 0$.

Table 3 shows the values of the momentum thickness at the outlet of the domain compared with the equivalent momentum thickness defined as $\theta_{D}=\left[\theta_{2}-\theta_{2}^{N} \tau^{G N}\right]=h C_{d} / 2$ which accounts for the contribution to the drag of the wall-friction. As one can see, $90 \%$ of the drag is determined by the momentum thickness at the outlet (which can be easily measured) hence, any possible variation on the modelling of the $\bar{\tau}_{w}(x)$ term will have a limited impact in the final determination of the drag. Note that the tests are conducted for a single velocity hence the variability of $C_{d}$ with $R e$ remains an open question. Nevertheless, we are in the fully turbulent regime, thus form drag coefficient is expected to remain constant for different $U_{\infty}$.

The drag coefficients for the various grids are shown in the last row of table 3. The Cro grid presents a 
slightly lower value of $C_{d}$ than the other three grids which show a $C_{d} \approx 0.44$. As mentioned in section 1 , very few studies are focused on low porosity grids, hence making comparisons with other authors difficult. As a first approach one can calculate the fence's drag based on the drag coefficient of individual bars; using this method and certain empirical correlations Hoerner (1965) estimates $0.5 \lesssim C_{d} \lesssim 0.6$. The same result is obtained by using the model proposed by Taylor (1963) (whose direct measurements by free fall in a water tank report $\left.C_{d} \approx 0.5\right)$. More recently Dong et al. (2008), using particle image velocimetry and the momentum equation, show $C_{d} \approx 0.5$. Alternatively, there are a large number of studies which, without presenting results for the present blockage $\sigma=30 \%$, show the trend followed by $C_{d}$ for different porosities. Without trying to provide an exhaustive review, results presented in Woodruff et al. (1963); Seginer (1972); Guan et al. (2003) are consistent with a drag coefficient between 0.4 and 0.6 for a $30 \%$ blockage.

There is approximately a $12 \%$ of difference between the drag coefficient of the Cro grid and the other three cases. It could be argued that this discrepancy is generated by the uncertainty associated to the method hypotheses. The described methodology relies on two assumptions, neglecting the pressure contribution and the approximation of the wall-friction by $\theta_{2}^{N} \tau^{G N}$. The former seems to hold equally well for the four grids (c.f. figure 1(a)) under boundary layer assumptions. However, $\bar{p}(y)=\bar{p}_{w}$ is only valid in the case $|\hat{v}| \ll 1$, which may not hold for every grid. In fact, (Rodríguez-López et al., 2016a) reported a negative mean vertical velocity persisting for a long streamwise extent downstream of large obstacles tripping a TBL. In the case $\hat{v}<0$, it would follow $\bar{p}_{w}>\bar{p}(y)$ which would entail an underestimation of the grid's drag. Following Rodríguez-López et al. (2016b,a), $\hat{v}<0$ would imply a larger interaction between inner and outer regions of the boundary layer. This is confirmed by the spectral measurements (presented in section 3.2) showing that there is a stronger link between inner and outer structures for the Cro grid which could imply that boundary layer assumptions are less applicable in this case and this therefore could explain, to some extent, the smaller drag of the Cro grid. With respect to the contribution of the shear stress, a different skin friction history for $x_{1}<x<x_{2}$ could change the value of the drag coefficient. However, $\theta_{2}-\theta_{D}$ seems to be reasonably constant for the four grids; which is in good agreement with the similar value of $\bar{\tau}_{w}(x)$ downstream of these grids shown in Table 2. An alternative explanation can be found by considering that the aspect ratio of the bars composing the Cro grid is smaller than those bars for the other grids (c.f. figure 2 and Table 1). This implies that their individual drag coefficient, and therefore the grid's drag would be also smaller (Hoerner, 1965). In practice, it is likely that both effects have a certain influence in the smaller drag of the Cro grid.

To summarize, the drag of the present fences is correctly estimated by $C_{d} \approx 0.45$ except for the Cro grid which appears to be $12 \%$ lower, most likely due to the appearance of non-zero vertical velocity and the smaller aspect ratio of its bars. This result is well within the experimental and theoretical scatter of results present in the literature. As has been reported before, we can conclude that integral properties of the grids are mainly dependent on their blockage with the differing distribution of porosity playing a secondary role. However, different sized bars do have a certain impact and they can play a more significant role in the turbulent properties immediately downstream of them and also influence the far-field development of the incipient TBL. These aspects are addressed in the next section where we will characterize the turbulence properties downstream 
1 of the various grids.

\subsection{Turbulence properties}

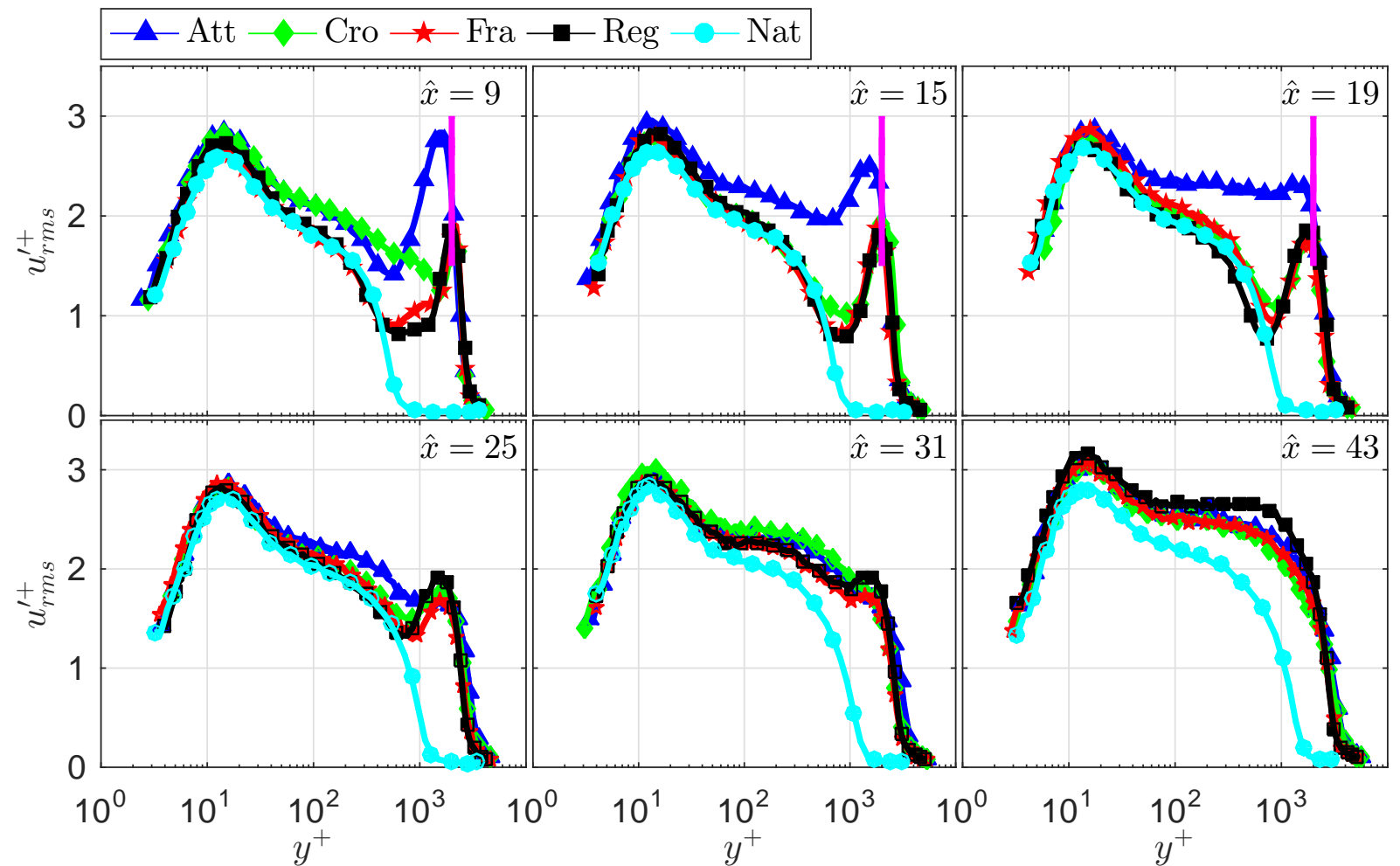

Figure 3: Profiles of inner-scaled turbulence level $u_{r m s}^{\prime+}$ for various grids and streamwise locations. The grids are always located at $x_{g}=100 \mathrm{~mm}$ where the incoming boundary layer thickness $\delta^{I} \approx 0$. The solid pink like shows the approximate location of the outer peak related with the shear layer.

The previous section has characterized the bulk behaviour of the grids using their drag coefficient. Despite 4 some differences for the Cro grid case, drag seems to be primarily determined by the porosity whereas its 5 distribution plays a secondary role. However, the turbulence characteristics are more likely to be influenced by the different grids' bars orientation and geometry. This section will show the one-point statistics and turbulence level in the lee of the various grids.

Figure 3 shows the profiles of $u_{r m s}^{\prime}$ for the various grids and streamwise locations. No correction is applied to mitigate the effect of limited spanwise resolution (downstream of the grids $u_{\tau}$ is smaller than in the natural case, hence $l_{w}^{+}$is also smaller and the resolution is improved). As a consequence, the peak turbulence intensity (at $\left.y^{+} \approx 15\right)$ increases slightly with streamwise distance for every case.

It seems logical to assume that the disturbed rms velocity profiles in the closest location to the grid will evolve with $\hat{x}$ until a closer resemblance to the natural case is achieved; indeed this situation is observed for every grid. In the closest measurement station $(\hat{x}=9)$ the profiles qualitatively follow the natural case in the inner region $\left(y^{+} \lesssim 300\right)$ whereas they display a clear departure further from the wall. A large peak is found in the external layer collapsing at $\hat{y}=1 \leftrightarrow y^{+} \approx 2000$ which is most likely due to the shear layer appearing between the grids' wake and the freestream. This peak decreases in magnitude with $\hat{x}$; simultaneously, the rms level in the region $300 \lesssim y^{+} \lesssim 1000$ increases with $\hat{x}$ for every grid. The interaction between the inner 


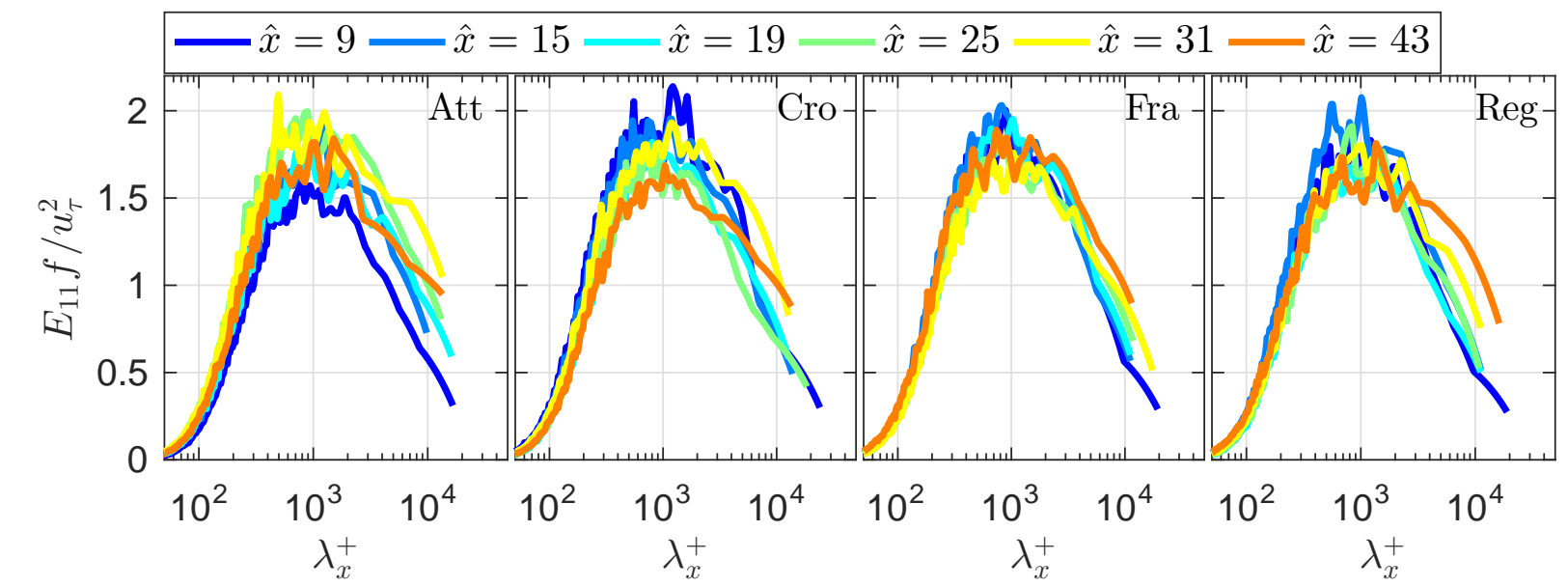

region and the grids' wake may have some similarities with a TBL flow developing under freestream turbulence. However, in the present case the shear layer also presents some influence in the near-wall region which would not be the case under freestream turbulent conditions. This topic will be further discussed in section 3.3.

Although the grids' wakes generate a highly turbulent fluid in their vicinity, these wakes meet and interact far upstream of the first measurement station where their turbulence intensity is significantly smaller. Turbulence decay downstream of single- and multi-scale grids has been broadly documented before (see, for instance Hurst and Vassilicos, 2007; Mazellier and Vassilicos, 2010). Considering the turbulence intensity downstream of gridgenerated turbulence, the turbulence level is reported to increase with the streamwise coordinate before reaching a peak at $x \approx 0.4 x^{\star}$; the turbulence intensity subsequently decreases further downstream. The definition of $x^{\star}=L_{0}^{2} / t_{0}$ as a relationship between the length and thickness of the largest bar appears when considering the meeting point of these bars' wakes. For the grid presenting the largest $x^{\star}=L_{0}^{2} / t_{0}=650 \mathrm{~mm}($ Fra $)$ and in the closest measurement point we have $\hat{x}=9 \leftrightarrow x=1.23 x^{\star}$; which is far downstream of the peak appearance. Contrastingly, the turbulence level of the shear layer is maintained significantly higher much further downstream, probably as a consequence of the high shear which sustains the turbulence production (see figure 5 and the discussion below).

Figure 4: Inner-scaled premultiplied spectra, $E_{11}\left(u^{\prime}\right) f / u_{\tau}^{2}$, as a function of inner-scaled length scale, $\lambda_{x}^{+}$, at $y^{+}=15$ for various grids and streamwise locations. The grids are always located at $x_{g}=100 \mathrm{~mm}$ where the incoming boundary layer thickness $\delta^{I} \approx 0$.

Summarizing, figure 3 at $\hat{x}=9$ shows an inner region $\left(y^{+} \lesssim 300\right)$ largely influenced by the presence of the wall and its mechanism of turbulence production for all grids; a second layer $\left(300 \lesssim y^{+} \lesssim 2000\right)$ related to the grids' bars' wakes which presents a lower intensity as a consequence of their progressive decay and a strong external peak related to the shear layer appearing above the grid. Further downstream, the different layers progressively mix with each other until a qualitative resemblance to the natural case is obtained. Further explanation can be sought in the study of the spectral content of the streamwise velocity fluctuations. If the inner region is actually dominated by the presence of the wall, then its spectra would be expected to resemble that of a natural TBL in this region.

Figure 4 shows the spectral content of the fluctuations for different grids and streamwise locations at $y^{+}=15$, i.e. the location of the inner peak of turbulent intensity. The spectra are plotted against the inner scaled length 
1 scale $\lambda_{x}^{+}=\lambda_{x} u_{\tau} / \nu=\bar{u}_{15} u_{\tau} /(f \nu)$, where the transformation from frequency to length has been performed based 2 on Taylor's hypothesis considering the mean velocity $\bar{u}_{15}=\bar{u}\left(y^{+}=15\right)$ at the inner peak location. It is clearly observed that the spectral energy at the inner peak is primarily concentrated around $\lambda_{x}^{+} \approx 1000$. This effect has 4 been broadly reported before as representative of the inner behaviour of natural TBLs (see e.g. Chernyshenko 5 and Baig, 2005b; Marusic et al., 2010; Rodríguez-López et al., 2016b). Note that the use of Taylor's hypothesis 6 in spatially evolving flows may be challenged; however, figures 3 and 4 show that the behaviour in the inner region seems to closely resemeble that of a natural TBL, hence the derivatives in the streamwise direction may be assumed to be sufficiently small so that Taylor's hypothesis may still be applied as commonly done in natural TBLs (e.g. Marusic et al., 2010).

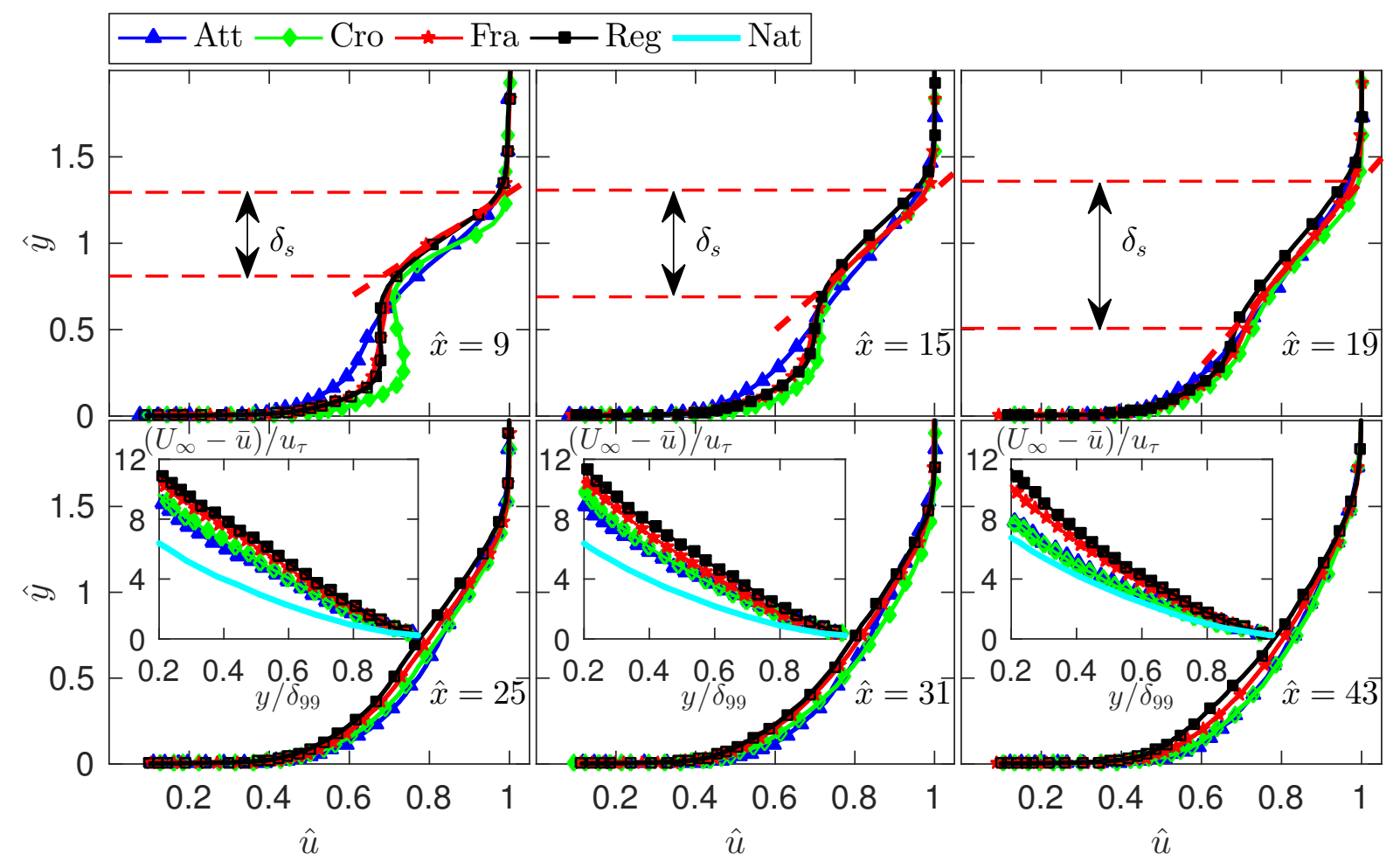

Figure 5: Wall-normal profiles of outer-scaled mean velocity $\hat{u}$ for various grids and streamwise locations. The dashed red lines in the upper three plots show the linear trends fitted to the shear layer along with their thickness $\delta_{s}$, for the Fra grid. The insets in the bottom row show the outer-scaled velocity defects profiles for the various grids. The grids are always located at $x_{g}=100 \mathrm{~mm}$ where the incoming boundary layer thickness $\delta^{I} \approx 0$.

In order to assess the relationship of the outer peak with the shear layer, one should compare its wall-normal extent with the thickness of the shear layer $\delta_{s}$. This thickness can be measured from the mean velocity profile by fitting a straight line in the vicinity of $\hat{y}=1$ (the central point of the shear layer). The point at which the mean velocity profile departs from the linear trend by a specified threshold (arbitrarily taken to be $0.02 U_{\infty}$ ) is defined as the lower limit of the shear layer which extends until $\delta_{99}$. Consequently, the distance between these two points is the thickness $\delta_{s}$. This is shown in figure 5 where the streamwise evolution of the mean profiles is studied. Whilst for the sake of brevity, the linear fit in the shear layer is only shown for the Fra grid; no qualitative changes are observed for the other grids. As expected, the thickness of the shear layer increases with the streamwise coordinate and, similarly, the intensity of the shear $(\mathrm{d} \bar{u} / \mathrm{d} y)$ decreases with $x$. For $\hat{x}>24$ 
1

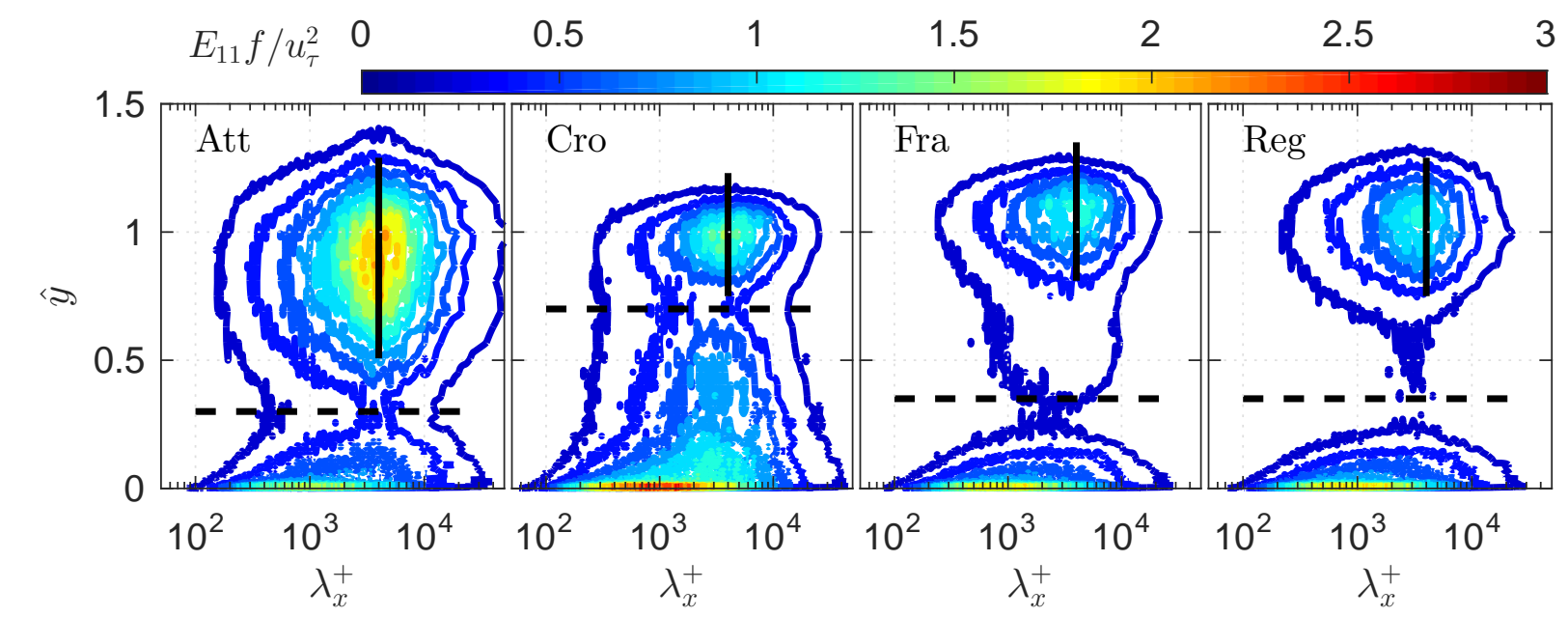

Velocity defect profiles are shown in the insets of figure 5 once the shear layer has lost its predominant effect (i.e. $\hat{x}>24$ ). Whilst the lack of collapse with the natural case is not surprising, there is a certain tendency for the velocity profiles downstream of the various grids to resemble the natural case more closely for increasing $x$. In essence, it seems reasonable that eddies in the outer region (scaling in outer units) need a longer streamwise distance (in physical units) to recover than significantly smaller scales near the wall (scaling in viscous units). This may be related with a larger turnover time associated with scales of the order $\delta_{99}$ rather than near-wall structures scaling with viscous units.

Figure 6: Inner-scaled premultiplied spectra, $E_{11}\left(u^{\prime}\right) f / u_{\tau}^{2}$, as a function of inner-scaled length scale, $\lambda_{x}^{+}$, and the wall-normal coordinate $\hat{y}$ for various grids and $\hat{x}=9$. Solid lines show the thickness of the shear layer $\delta_{s}$ and dashed lines are provisionally sketched separating inner and shear layers. The grids are always located at $x_{g}=100 \mathrm{~mm}$ where the incoming boundary layer thickness $\delta^{I} \approx 0$.

Figure 6 shows the wall-normal contours of premultiplied spectra $E_{11}\left(u^{\prime}\right) f / u_{\tau}^{2}$, as a function of inner-scaled length scale, $\lambda_{x}^{+}$at the first measurement station $(\hat{x}=9)$. The scaling of the inner peak is solely influenced by the wall with a concentration of spectral energy around $\lambda_{x}^{+} \approx 1000$ as confirmed in figure 4 . The outer peak appearing in the $u_{r m s}^{\prime}$ profiles is also clearly present in the spectral contours. Moreover, its wall-normal position and extent correspond with the thickness of the shear layer $\delta_{s}$ estimated as described above. Note that $\delta_{s}$ is defined by means of the departure from a linear trend in the mean profile and it is a valid scaling for the size of the spectral peak. This establishes a link between the shear in this region (which entails an enhanced production level) and the spectral content. For every grid, it is also clear that the intermediate region between the inner and outer peaks contains a much smaller turbulent kinetic energy as a consequence of the grids' wake having decayed much faster in the absence of any mean shear which sustains the turbulence production.

Special mention is required for the Att grid which, as opposed to the other three, shows a broader peak in the shear layer with a larger energy content which also extends for a greater wall-normal extent. This is probably due to a combination of much thicker vertical bars and open gaps whose interactions enhance the turbulent activity in the shear layer of this grid. Contrastingly, Fra and Reg grids present a narrower peak in the shear region which is undoubtedly separated from the near-wall region. This result could be predicted 
1 by looking at figure 3 in $\hat{x}=9$ where the rms profiles for these two grids exactly follow the natural case for

$y^{+}<300$ as opposed to the Att case and, to a lesser extent, the Cro for which there is a larger departure.

The large wall-normal extent and energy content of the shear layer for the Att grid may influence the near-wall

4 behaviour in a different way than the other two grids. In fact, this was reported in Rodríguez-López et al.

5 (2017) where the Att grid was observed to present up to a $10 \%$ larger wall shear stress fluctuations $\left(\tau_{w, r m s}^{\prime+}\right)$

6 than that predicted at a comparable Reynolds number. This effect is likely due to the enhanced interaction between inner and outer layers as a consequence of its larger energy content.

As opposed to the other 3 grids, Cro case presents a larger energy content in the region $0.2 \leq \hat{y} \leq 0.6$. 9 Although this extra energy seems to be relatively independent from the shear layer; it may be assumed that it does not come from the wall either (the small streamwise location, $\hat{x}=9$, makes it unlikely for the boundary layer to have grown up to $\hat{y} \approx 0.6)$. Hence it seems logical to hypothesize that, for the Cro case, the grid's wake persist for a longer streamwise distance or, at least, presents a larger influence in the near-wall region. This may have some implications on other results, for instance, Rodríguez-López et al. (2016a) related a larger influence of the wake on the wall with the presence of negative wall-normal velocities. This was previously hypothesized in section 3.1 as a candidate for explaining the slightly different value of $C_{d}$ in the Cro case.

Summarizing, the spectral content and rms profiles provide enough evidence to suggest the appearance of two well-differentiated layers: (i) a wall layer as a consequence of the interaction with the wall and (ii) a second region related with the shear layer appearing between the grid and the freestream with a large turbulence activity due to the production facilitated by the high mean shear. Furthermore, one can tentatively sketch a qualitative separation between these two layers where the spectral contours are narrower (or even disappearing such as the Reg case). This separation is shown in figure 6 with a horizontal dashed line. Section 3.3 will propose a systematic method to establish a way to measure the separation between these layers which may have some implications for boundary layer scaling.

\subsection{Two layers concept}

The appearance of two layers inside the TBL such as those characterized in section 3.2 has been previously reported by different authors. One example of these layers, appears downstream of a sudden change in surface roughness. In these cases two layers are usually differentiated: one layer located far from the wall (whose properties are set by the rough upstream boundary conditions) and a second region located close to the wall (with properties set by the roughness condition dowstream of the sudden change). With the streamwise distance, this second region progressively grows by mixing with the outer one in order to adapt to the new boundary condition. Several examples can be found to establish a well-defined border between these two layers: for instance, Andreopoulos and Wood (1982) propose successive streamwise differentiation of velocity profiles. Contrarily, Antonia and Luxton (1971) showed that the border between these two layers could be characterized by an inflection in the profile $\hat{u} \propto \sqrt{y}$. More recently, this procedure was employed by Hanson and Ganapathisubramani (2016) who additionally proposed different velocity scalings for the inner and outer layers. A further 
methodology is proposed by Efros and Krogstad (2011), who fitted straight lines to the $u_{r m s}^{\prime+}$ profiles.

Apart from studies considering step changes in surface roughness; the appearance of two well-differentiated layers has also been identified in TBLs subject to freestream turbulence (Dogan et al., 2016) or evolution from strong tripping conditions (Rodríguez-López et al., 2016a). In this case, Rodríguez-López et al. (2016a) who defined the border between them based on a drop in the correlation level with the near-wall region below a certain threshold.

The main objective of this section is to propose and validate a method being able of distinguish between these two regions such that it should establish a clear border between them. Furthermore, it is conjectured that the thickness of the inner layer would act as an effective "outer scale" for the pseudo-TBL development underneath and therefore several quantities could be found to scale with this thickness. A qualitative sketch of the two layers is presented in figure $7(\mathrm{a})$. For completeness, the shear layer and its thickness $\delta_{s}$ described in section 3.2 are also sketched.

However, none of the aforementioned methods of internal layer detection can be applied, essentially because the flow configuration is radically different. An alternative method therefore is proposed which can be applied to the present flow. Given that the appearance of the two layers has been postulated by studying the velocity fluctuations and spectral profiles, a deeper study of the $u_{r m s}^{++}$profile seems appropriate. In fact, note that these profiles (for the Fra and Reg cases) follow the natural TBL trend with surprising accuracy, despite the much lower Reynolds number of this case. This suggests that, in essence, one could look for a natural boundary layer of a Reynolds number smaller than the Grid+TBL case which presents the same $u_{r m s}^{\prime+}$ profile in the inner region. However, this procedure (finding a natural case that resembles the measured profile close to the wall) requires an underlying assumption that may be contradictory, namely, it neglects the influence of the outer region on the inner layer. Whilst there is experimental evidence showing that this interaction does exist (especially the influence of freestream turbulence on TBLs); we might neglect it as a first approach according to the results presented in figure 6. One can easily observe that there is indeed a much larger separation, at least for the Fra and Reg cases, than the typical interaction between freestream turbulence and TBLs (see, for instance Dogan et al., 2016). On the other hand, some problems may be foreseen in the Att and Cro cases due to the insufficient separation of layers or an excessive influence of the outer layer in the near-wall region (c.f. figure 6).

\begin{tabular}{|c|c|c|c|c|c|c|}
\hline & Symbol & Reference & $\min R e_{\tau}$ & $\max R e_{\tau}$ & Points & Type \\
\hline OS13 & $\boldsymbol{\Delta}$ & Orlü and Schlatter (2013) & 794 & 2017 & 14 & Exp (HW) \\
\hline DE00 & $\boldsymbol{\Delta}$ & Degraa et al. (1999) & 541 & 10023 & 5 & Exp (LDV) \\
\hline HNMC09 & $\boldsymbol{V}$ & Hutchins et al. (2009) & 2820 & 18830 & 5 & Exp (HW) \\
\hline RBB16 & $\boldsymbol{\nabla}$ & Rodríguez-López et al. (2016b) & 285 & 1058 & 7 & Exp (HW) \\
\hline SJM13 & $\diamond$ & Sillero et al. (2013) & 1307 & 1989 & 6 & DNS \\
\hline SO10 & $\square$ & Schlatter and Örlü (2010) & 252 & 1271 & 10 & DNS \\
\hline DLV17 & $\star$ & Díaz-Daniel et al. (2017) & 400 & 650 & 6 & DNS \\
\hline
\end{tabular}

Table 4: Summary of experiments (Exp) and direct numerical simulations (DNS) used to fit the expression $u_{r m s}^{\prime+}=f\left(R e_{\tau}\right)$ given by equation (2). 


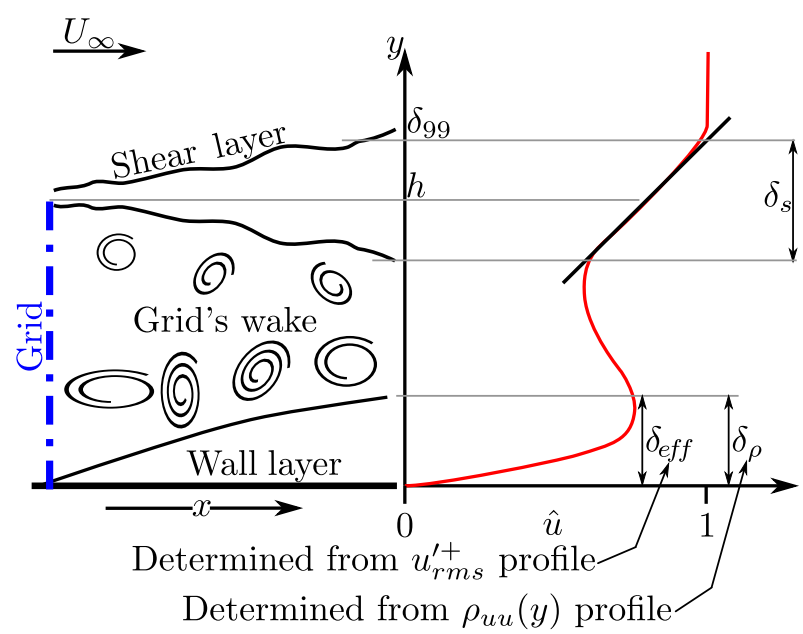

(a)

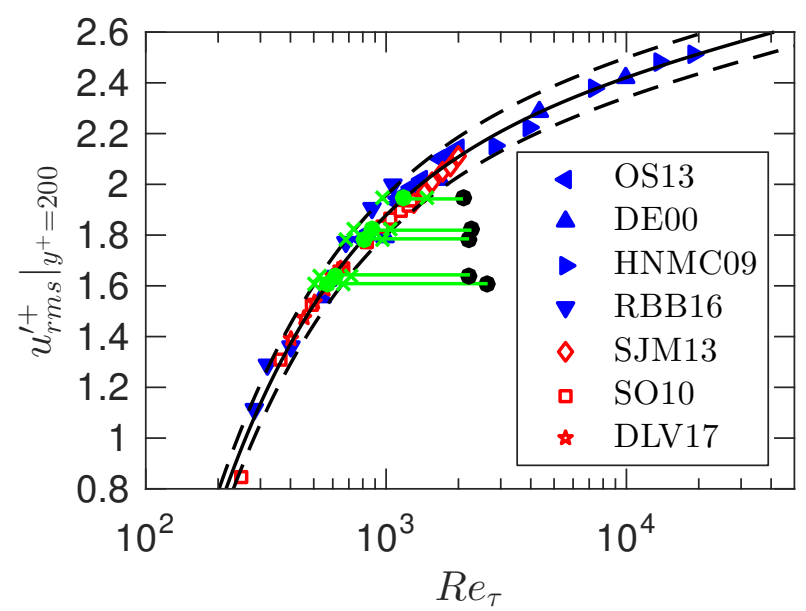

(b)

Figure 7: (a) Sketch of the $\delta_{\text {eff }}$ concept, the boundary layer properties are only influenced by the wall layer setting $\delta_{e f f}$ or $\delta_{\rho}$ as the typical length scale. (b) Compilation of $u_{r m s}^{\prime+}$ at $y^{+}=200$ for different experiments (filled triangles) and DNS simulations (empty symbols); see table 4 for the interpretation of the legend. The black dots show the $R e_{\tau}$ for $R e g$ grid at various $x$ based on $\delta_{99}$ and the green dots show their corresponding $R e_{e f f}=\delta_{e f f} u_{\tau} / \nu$. The solid line is the empirical fitting given by equation (2) and the dashed lines show the $95 \%$ confidence interval.

\subsection{1 $\delta_{\text {eff }}$ definition}

$2 \delta_{\text {eff }}$ is defined as the thickness of an hypothetical natural TBL which would provide the same level of $u_{r m s}^{\prime+}$ 3 in the wall layer of the Grid+TBL cases. The first problem to consider is which wall-normal extent should be 4 compared with the natural case. The very near-wall region (buffer layer and below) is uniquely determined by 5 the presence of the wall and its boundary condition, hence a resemblance with the natural case is expected and very little information could be extracted from this fact. Therefore, comparison with the natural case should be sought further away from the wall and $y^{+}=O(100)$ seems a good starting point.

The procedure is shown in figure 7(b) and described as follows: (i) compile the values of $u_{r m s}^{+}$at $y^{+}=$ 100, 200, 300 for several experiments and direct numerical simulations covering two decades of $R e_{\tau}$ (blue and red symbols in figure $7(\mathrm{~b})$ ). (ii) fit an analytical expression $\left.u_{r m s}^{\prime+}\right|_{y^{+}=i}=f_{i}\left(R e_{\tau}\right)$ with $i=100,200,300$ (solid black line in figure $7(\mathrm{~b}))$. (iii) Use the measured level of $u_{r m s, i}^{\prime+}$ at the three wall normal locations to find $R e_{\tau}^{e f f, i}$ such that $\left.u_{r m s, i}^{\prime+}\right|_{y^{+}=i}=f_{i}\left(R e_{\tau}^{e f f, i}\right)$. As shown in figure $7(\mathrm{~b})$ with black dots the Reynolds number based on $\delta_{99}$ does not capture the phenomena hence we find the "effective" Reynolds which should have the same level of fluctuations at that particular $y^{+}$(i.e. the green dots in figure 7(b)). (iv) The effective Reynolds number would be the average between the results obtained at the three wall-normal locations $\left(\operatorname{Re} e_{\tau}^{e f f}=\sum R e_{\tau}^{e f f, i} / 3\right)$. (v) Thus the thickness of the inner layer (so-called effective thickness) would be $\delta_{\text {eff }}=\operatorname{Re}_{\tau}^{e f f} \nu / u_{\tau}$. The functional form of $f_{i}\left(R e_{\tau}\right)$ is postulated as

$$
\left.u_{r m s}^{\prime+}\right|_{y^{+}=i}=A_{i} R e_{\tau}+B_{i} \log R e_{\tau}+C_{i} \frac{\log R e_{\tau}}{R e_{\tau}}
$$

where the selection of the functional form is not intended to provide any physical explanation of the phenomena involved. The functional dependence proposed by Marusic and Kunkel (2003) could not be employed since it is 
applicable only for wall-normal positions farther from the wall $\left(y^{+}>300\right)$. Furthermore, scaling based on the diagnostic plot (Alfredsson et al., 2011) was avoided since it does not provide any direct relationship with the boundary layer thickness.

Figure 7(b) shows one example of this procedure (Reg grid at $\left.y^{+}=200\right)$, other examples are similar and they are not shown for brevity. In this figure, one can observe the accurate fit provided by equation (2) to the various experiments and simulations of natural TBLs. Note that no correction for limited spanwise resolution has been applied to any data since this effect is expected to be negligibly small at $y^{+}>100$ (Hutchins et al., 2009). Note also that, due to the lack of experimental data at very high $R e_{\tau}$, the uncertainty (given by the $95 \%$ confidence interval) in this region is larger. This may present a problem for the furthest $\hat{x}$ locations of the experiment where $R e_{\tau}^{e f f} \rightarrow R e_{\tau}=\delta_{99} u_{\tau} / \nu \approx 3000$. The solid black dots in figure 7(b) show the Reynolds number based on $\delta_{99}$; whereas the green dots show the value of $R e_{\tau}^{e f f}$ estimated from the measured level of $u_{r m s}^{\prime+}$. It is clearly observed that the original value of $R e_{\tau}$ is not the correct one for scaling the turbulence intensity at this point.

\subsection{2 $\delta_{\rho}$ definition}

Alternatively, one can follow the methodology proposed by Rodríguez-López et al. (2016a) who defined the wall layer as the region in which the correlation of the streamwise velocity fluctuations with a point located in the buffer layer was larger than a certain threshold $\rho_{u u}^{\star}=0.3$. Analogously, two-point streamwise velocity correlations were performed as described in section 2.3. The wall hot wire was located at a fixed point $\left(x_{0}, y_{0}^{+} \approx\right.$ $\left.4, z_{0}\right)$ and the free hot wire was traversed in the wall-normal direction for the same $x_{0}$ and $z_{0}$. To account for the inclination of the turbulent structures a time delay $\eta$, between the two signals was applied following extensive experimental evidence (Brown and Thomas, 1977; Robinson, 1986; Boppe et al., 1999; Marusic and Heuer, 2007). In these conditions the correlation factor can be defined as

$$
\rho_{u u}(y)=\frac{\int_{0}^{\infty} u_{w h w}^{\prime}\left(y_{0}, t+\eta\right) u_{f h w}^{\prime}(y, t) \mathrm{d} t}{u_{w h w, r m s}^{\prime} u_{f h w, r m s}^{\prime}} .
$$

The point $y_{\star}$ at which $\rho_{u u}\left(y_{\star}\right)<\rho_{u u}^{\star}=0.3$ can be taken as representative of the inner layer. For a natural boundary layer this occurs at approximately $y / \delta \approx 1 / 8$ (scaling with outer units, see e.g. Marusic and Heuer, 2007; Rodríguez-López et al., 2016b; Díaz-Daniel et al., 2017). Since the objective is obtaining the thickness of a natural boundary layer which would exhibit similar near-wall behaviour, we can define an alternative definition of the effective thickness as $\delta_{\rho}=8 y_{\star}$, where the subindex $\rho$ symbolizes that it has been obtained from the wall-normal profiles of streamwise correlation. The factor of 8 is dependent on $y_{0}$, the wall-normal position of the WHW. Note however, that it does not play any role concerning the validity of $\delta_{\rho}$ as a scaling parameter since this is invariant to any multiplicative constant. 


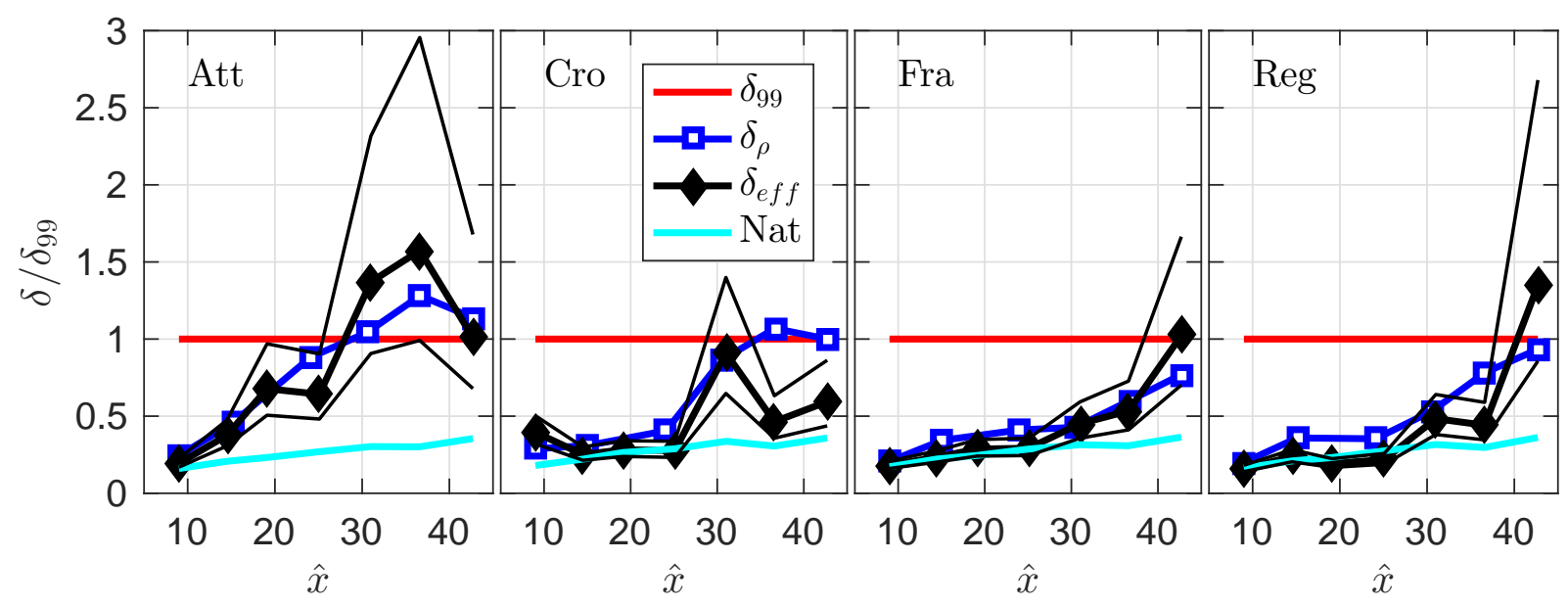

Figure 8: Evolution of the effective thickness $\delta_{\text {eff }}$ (with $95 \%$ confidence bounds) and the thickness based on the correlation level $\delta_{\rho}$ for various grids. The grids are always located at $x_{g}=100 \mathrm{~mm}$ where the incoming boundary layer thickness $\delta^{I} \approx 0$. The solid light-blue line shows the value of the natural TBL thickness $\left(\delta_{99}^{N a t}\right)$ for the various streamwise locations.

\subsection{3 $\delta_{\text {eff }}$ and $\delta_{\rho}$ evolution}

2 Figure 8 shows the streamwise evolution of these two definitions of effective thickness for the various grids 3 tested. The $95 \%$ confidence interval in the determination of $\delta_{\text {eff }}$ (the two solid thin lines in figure 8 ) is the 4 same as shown in figure 7(b). This is larger for high Re since there are less experiments at these conditions.

${ }_{5}$ Furthermore, the logarithmic trend of the curve in equation (2) makes it harder to extrapolate large $R e_{\tau}^{e f f}$

6 from the turbulence intensity profile. The agreement between the two definitions of the inner layer thickness is

7 clear and therefore it suggests the appearance of a well-defined physical mechanism in this region. The overall

8 behaviour for all the grids is as expected, $\delta_{\text {eff }}$ is much smaller than $\delta_{99}$ close to the fences and grows by mixing 9 with the grids' wake and the shear layer until all the turbulent flow interacts with the near-wall region in the 10 same manner as a TBL would do. The fact that $\delta_{\text {eff }}$ or $\delta_{\rho}$ can be larger than $\delta_{99}$ for the furthest streamwise locations may be possible because of the influence of the turbulent outer fluid. In fact, a similar effect is observed for TBLs developing under the influence of freestream turbulence (Dogan et al., 2016) where certain properties of the TBL (such as the departure from the logarithmic law) are shown to be representative of a 14 thicker boundary layer. Essentially, the same trend is observed here (especially for the Att grid), due to the large energy in the outer part of the TBL, the near-wall region behaves as a natural TBL which would have a thickness larger than $\delta_{99}$. Therefore these methods, present the potential of detecting not only the thickness of the wall layer but also the effective thickness generated as a consequence of an enhanced influence of the wake into the near-wall region.

To summarize, after the identification of two differentiated layers in the grid+TBL flow in section 3.2 ; this section has presented two systematic procedures by which one can establish the thickness of the inner layer based on the $u_{r m s}^{++}$profile $\left(\delta_{e f f}\right)$ or the wall-normal variation of streamwise velocity correlation $\left(\delta_{\rho}\right)$. 


\subsection{Scaling of wall shear stress fluctuations}

2 The first test that these scalings should fulfil is the correct determination of the behaviour near the wall. A 3 possible way to assess this behaviour is by means of the fluctuating wall shear stress $\tau_{w, r m s}^{\prime+}$ (Schlatter and Örlü, 4 2010, 2012). This is shown to present a logarithmic trend with $R e_{\tau}$ (which depends on the thickness of the 5 boundary layer), hence it has potential as a diagnosis quantity for this new scaling. Rodríguez-López et al. 6 (2017) obtained the fluctuations of the wall shear stress by means of a wall-mounted hot wire located in the 7 inner region. This hot wire was calibrated against a naturally growing TBL where the wall shear stress can s be extrapolated from the mean velocity profile as shown in Rodríguez-López et al. (2015). They showed that $\tau_{w, r m s}^{\prime+}$ in the wake of these porous fences presents an adaptation region in which $\tau_{w, r m s}^{\prime+}$ was significantly lower than the predictions, followed by a tendency to recover the expected value. The hypothesis is that only the inner layer (of thicknes $\delta_{\text {eff }}$ or $\delta_{\rho}$ ) contributes to those fluctuations. From there, it follows that the so-called adaptation region in Rodríguez-López et al. (2017) was just the growth of the inner layer which progressively resembles $\delta_{99}$ with the streamwise distance as shown in figure 8 . This is therefore consistent with a two-layer structure in which the wall layer grows with $x$ until the resemblance of a TBL since $R e_{\tau}^{e f f} \rightarrow R e_{\tau}$ for large $x$.

Figure 9 presents the same results of $\tau_{w, r m s}^{\prime+}$ but now scaled with the effective thickness of the inner layer calculated by the aforementioned procedures. The first result is the small dispersion appearing between $\delta_{e f f}$ calculated at the three wall-normal locations. This result is satisfactory since it ensures that the correct flow phenomena are captured at various wall-normal locations. Furthermore, it is strikingly clear that the so-called adaptation region from the grids' disturbances presents a well-defined scaling and it is not just a simple arbitrary transient process to adapt to the new boundary conditions. Similarly, the thickness of the wall layer obtained by means of the correlation level also presents a clear agreement with $\delta_{\text {eff }}$ (as could be anticipated by their similarity in figure 8). Moreover, it is also a valid scaling parameter for the wall shear stress fluctuations. A side note is required for the $A t t$ case. The new scaling significantly improves the data's trend $\left(\tau_{w, r m s}^{\prime+} \propto \log R e_{\tau}^{e f f}\right)$. However, the values appear to be shifted by a constant of approximately 0.025 with respect to the prediction of Schlatter and Örlü (2010). It is hypothesized that this could be related with the extraordinarily high intensity of the shear layer peak (discussed above in section 3.2) which enhances the fluctuations in the nearwall vicinity above the expected natural trend. However, the fact that the new scaling recovers the correct trend $\left(\tau_{w, r m s}^{\prime+} \propto \log R e_{\tau}\right)$ supports the use of this novel scaling for disrupted boundary layers.

Whereas the correlation proposed by Schlatter and Örlü (2010) (based on DNS data, exempt from any measurement bias) is $\tau_{w, r m s}^{\prime+}=0.298+0.018 \log R e_{\tau}$; Mathis et al. (2013) proposed shifting it by a constant $\tau_{w, r m s}^{\prime+}=0.245+0.018 \log R e_{\tau}$ to account for a damping in the fluctuations due to the interaction between the hot-wire thermal wake and the wall substrate (Brunn, 1995; Chew et al., 1998). This attenuation appears independently of spanwise resolution effects (which are corrected in the present measurements as shown in Rodríguez-López et al. 2017). In any case, $\tau_{w, r m s}^{\prime+}=A+B \log R e_{\tau}$ can be interpreted as a constant level of fluctuations (likely caused by the presence of streaks Chernyshenko and Baig 2005a) plus a weak influence of the outer fluid (scaling with $\log R e_{\tau}$ ). It has been reported that this interaction can be generated by a superposition 


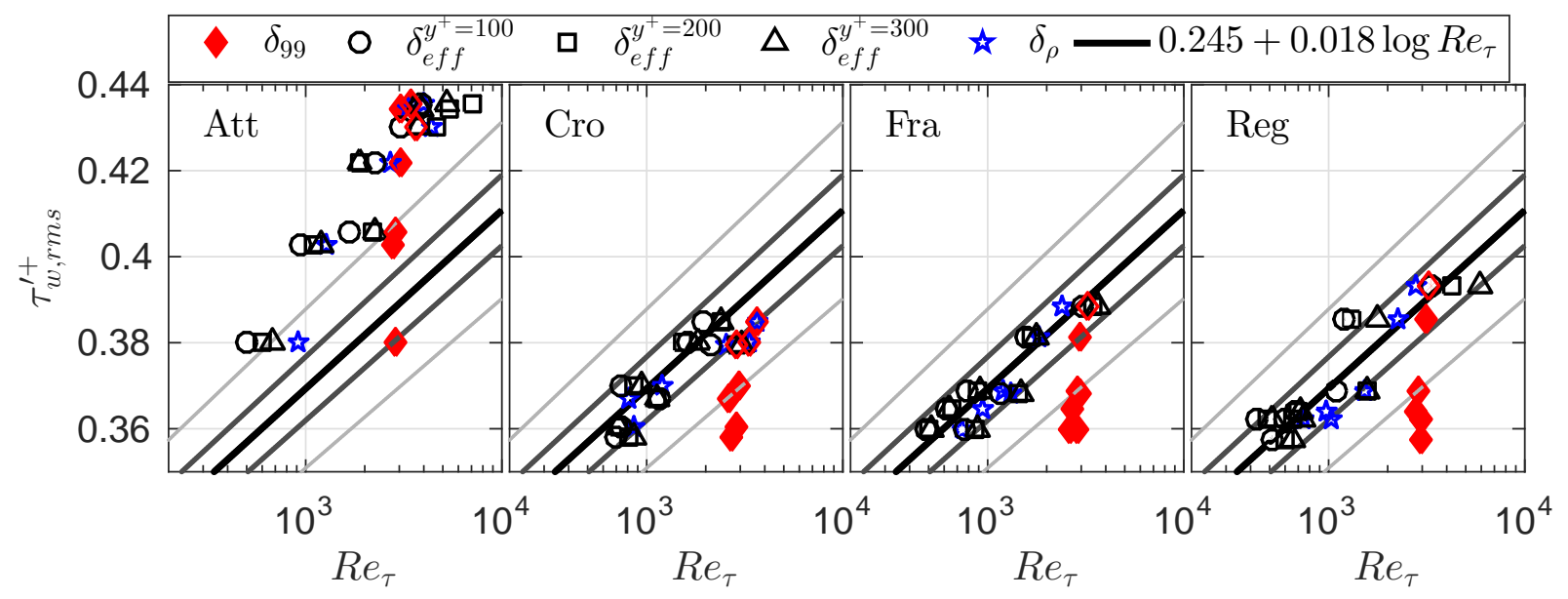

Figure 9: Wall shear stress fluctuations, $\tau_{w, r m s}^{\prime+}$, as a function of $R e_{\tau}$ based on different definitions of $\delta$ for various grids and streamwise locations. Data is taken from Rodríguez-López et al. (2017). Solid lines show $\tau_{w, r m s}^{\prime+}=0.245+0.018 \log R e_{\tau}$ with $2 \%$ and $5 \%$ margins. The grids are always located at $x_{g}=100 \mathrm{~mm}$ where the incoming boundary layer thickness $\delta^{I} \approx 0$.

and a modulation of the small near-wall structures by the large scale turbulence in the logarithmic layer (e.g. Mathis et al., 2011). Recovering $\tau_{w, r m s}^{\prime+} \propto \log R e_{\tau}$ suggests that the physics of the flow in terms of the outerinner interaction are captured by the scaling but the intensity of this effect is underestimated. This could be a consequence of a distinct influence of the grid's wake on the wall region as hypothesized in section 3.2. An alternative interpretation of the distinct behaviour of Att grid could be given by the procedure described in Hanson and Ganapathisubramani (2016). In it, they specify a distinct velocity scale for the wall layer rather than $u_{\tau}$ which would then recover the trend proposed by Schlatter and Örlü (2010). However this would be at odds with figures 3 and 4 which show $u_{\tau}$ to be an adequate scaling for the location and magnitude of the inner turbulence peak. It also seems reasonable to assume that the skin-friction fluctuations scale with the local shear rather than with an outer-scale velocity such as $U_{\infty}$.

By recovering the expected trend of $\tau_{w, r m s}^{\prime+}$, this section has shown that the effective thickness of the inner layer is valid as an outer scaling for the pseudo-TBL developing underneath grid generated turbulence. The appearance of this scaling poses new questions regarding its applicability to other outer-scaled TBL phenomena. The next sections therefore will study the different length scales present in the flow and how their evolution can be explained in terms of this new scaling.

\section{$3.5 \quad$ Integral length scale, $L_{11}$}

After having set the basis for the new scaling based on $\delta_{e f f}$, this section will study the wall-normal profiles of integral length scale and how their scaling may differ from that of a natural TBL. The longitudinal integral length scale $L_{11}$ is obtained from single-component single-point hot-wire anemometry by considering the integral time scale $\mathcal{T}_{11}$ and the convection velocity $u_{c}: L_{11}=\mathcal{T}_{11} u_{c}$. Several studies have been focused on obtaining a value for the mean convection velocity across the TBL (Del Alamo and Jimenez, 2009; Geng et al., 2015) concluding that one can consider as a first approach the mean velocity profile $u_{c}=\bar{u}(y)$ (i.e. Taylor's hypothesis) for wall-normal positions such that the mean velocity is $\bar{u}^{+} \gtrsim 11$. Considering the autocorrelation function 
${ }_{1} \rho_{u u}(\eta)=\left(\int u^{\prime}(t) u^{\prime}(t+\eta) \mathrm{d} t\right) /\left(u_{r m s}^{\prime 2}\right)$, one can obtain $\mathcal{T}_{11}$ by numerical integration of $\rho_{u u}(\eta)$. In order to 2 avoid the noise appearing at low $\rho_{u u}$, or equivalently, large time delays $\eta$, one can fit an exponential decay for ${ }_{3} \rho_{u u}<0.5$. Calculation of $\mathcal{T}_{11}$ based on Fourier spectra (not shown) provided qualitatively the same results.
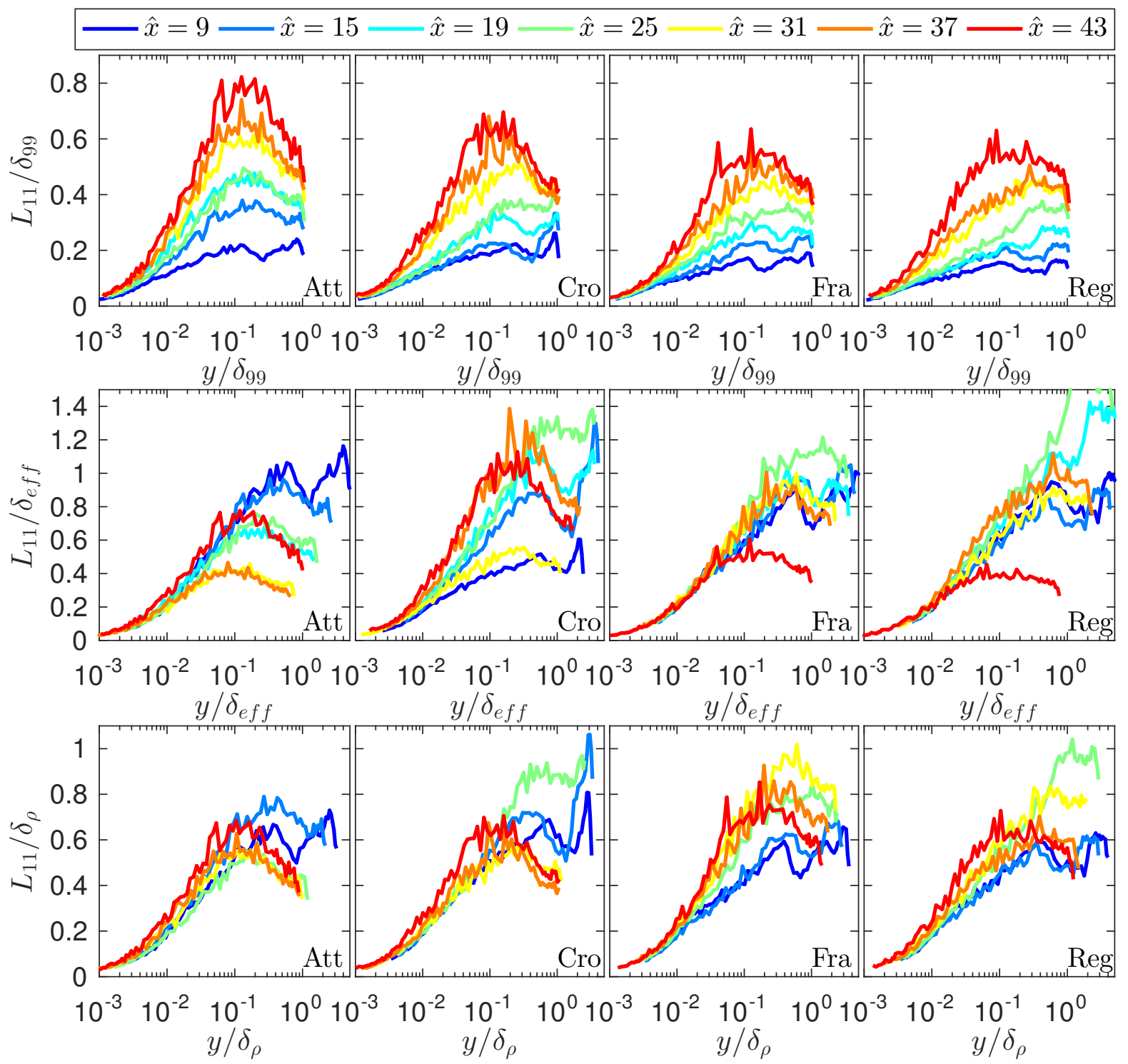

Figure 10: Profiles of integral length scale $L_{11}$, for various grids and streamwise locations normalized with $\delta_{99}$ (first row) and with $\delta_{\text {eff }}$ (second row) and $\delta_{\rho}$ (third row). The grids are always located at $x_{g}=100 \mathrm{~mm}$ where the incoming boundary layer thickness $\delta^{I} \approx 0$.

The first row of figure 10 shows the profiles of $L_{11}$ scaled with $\delta_{99}$. Note that $\delta_{99}$ includes the three-layer 5 structure described above, namely, wall layer, grid wake and shear layer. However, the typical length scales 6 in the near-wall region should be expected to scale with the thickness of the wall layer rather than with $\delta_{99}$. 7 Consequently, no collapse is observed in the first row of figure 10 for any grid or streamwise location. If, in the 8 contrary, we use a scaling which accounts only for the thickness of the wall layer, that is, $\delta_{\text {eff }}$ or $\delta_{\rho}$ as proposed 9 above. Then $L_{11}$ is shown to collapse when scaled with those parameters in the near wall region (second and 10 third rows of figure 10 for $y / \delta \lesssim 0.2$ ). Logically, this scaling is not valid out of the wall layer since it does not 11 capture the physics there, which do not depend on the thickness of the near-wall region.

A few differences can be observed between the two proposed scaling parameters. For cases where the 
1

2

spectral separation of scales is less clear (Att and Cro grids), $L_{11}$ seems to scale better with $\delta_{\rho}$ rather than $\delta_{\text {eff }}$. This is likely due to the methodology followed to obtain $\delta_{\text {eff }}$ which relies on a sufficient separation of the spectral behaviour. Contrastingly, for cases where the separation in the turbulence intensity profiles is larger, the definition of $\delta_{\text {eff }}$ provides a better collapse. Independently, note that both $\delta_{e f f}$ and $\delta_{\rho}$ are more valid as a scaling parameter for the outer region of the wall-bounded flow than $\delta_{99}$ which does not reflect the separation between the wall and wake layers.

For Fra and Reg cases the furthest streamwise location $(\hat{x}=43)$ presents a distinct behaviour when scaled with $\delta_{e f f}$. Note that this is likely due to the large uncertainty in the determination of $\delta_{e f f}$ for large $R e_{\tau}$ values. In fact, figure 8 has shown that the point located at $\hat{x}=43$ presents an uncertainty of approximately $50 \%$ which, in turn, translates into a dubious scaling for the last point. If, on the other hand, we consider smaller streamwise locations (or, equivalently, Reynolds numbers), the collapse is shown to hold up to $y / \delta_{\text {eff }}$ of order unity.

Note also that Att case presents as good a collapse as Fra and Reg grids despite its differences in terms of $\tau_{w, r m s}^{\prime+}$ (c.f. figure 9). In fact, Rodríguez-López et al. (2017) reported that, despite its larger $\tau_{w, r m s}^{\prime+}$ value, the autocorrelation of the wall shear stress for the Att grid was dominated by the presence of streaks (as in the natural case). This fact could be linked with the recovery of the trend $\tau_{w, r m s}^{\prime+} \propto \log R e_{\tau}$ which was also related with the presence of streaks. In light of this evidence, it seems reasonable to hypothesize that the large turbulent kinetic energy in the outer part of the Att grid's wake (generated by the combination of wakes of thick bars and large open areas) modifies the near-wall region by modifying its upper boundary condition. That is, the flow in the wall region, characterized by $\delta_{e f f}$, perceives the wake of the grid as its upper limit rather than the freestream. Since in this particular grid, the wake is more highly energetic (c.f. for instance, figure 6), the fluctuations are enhanced. However, the formation mechanisms of turbulence in the wall region (streaks) and its length scales remain unaltered. In essence, this mechanism would be equivalent to an inner boundary layer of thickness $\delta_{\text {eff }}$ in which the apparent freestream is modified by the presence of the grid's wake enhancing turbulent fluctuations in a broadband manner, thus not modifying the predominant $L_{11}$.

To summarize, the integral length scale has been obtained from the correlation function and Taylor's hypothesis showing that for a natural TBL it collapses in the inner region followed by a plateau with a Reynolds trend in the outer region. For the disrupted cases, $\delta_{99}$ cannot be used as a scaling parameter since it does not reflect the physics of the flow in the inner region which depend on its local thickness (determined either by $\delta_{e f f}$ or $\delta_{\rho}$ ). Use of this scaling enables the collapse of $L_{11}$ across the inner layer. For cases with insufficient spectral separation between the layers (Att and Cro grids as described in section 3.3), $\delta_{\rho}$ presents a better collapse of the $L_{11}$ profiles. Alternatively, if the spectral separation is larger (Fra and Reg grids) $\delta_{e f f}$ is a better scaling parameter except for very high $R e_{\tau}$ due to the larger uncertainty at the last position. 


\subsection{Spanwise turbulent structure}

2 The streamwise characteristic lengths of the flow have been studied by means of $L_{11}$ in section 3.5. However, the 3 flow in the vicinity of a wall is strongly anisotropic in the spanwise direction. The spanwise organization of TBL 4 flows in the near-wall region is characterized by the appearance of large or very large meandering structures 5 resident in the logarithmic layer (e.g. Ganapathisubramani et al., 2005; Hutchins and Marusic, 2007a). These structures have also been reported to appear downstream of mild disruptions such as strong tripping conditions 7 (Rodríguez-López et al., 2016b). Moreover, they also imprint their characteristics on the near wall region as a consequence of the inner-outer interaction mechanisms (Hutchins and Marusic, 2007b). As reported in Hutchins and Marusic (2007a), the spanwise separation of these structures clearly scales with the local boundary layer new definitions of the internal thickness.
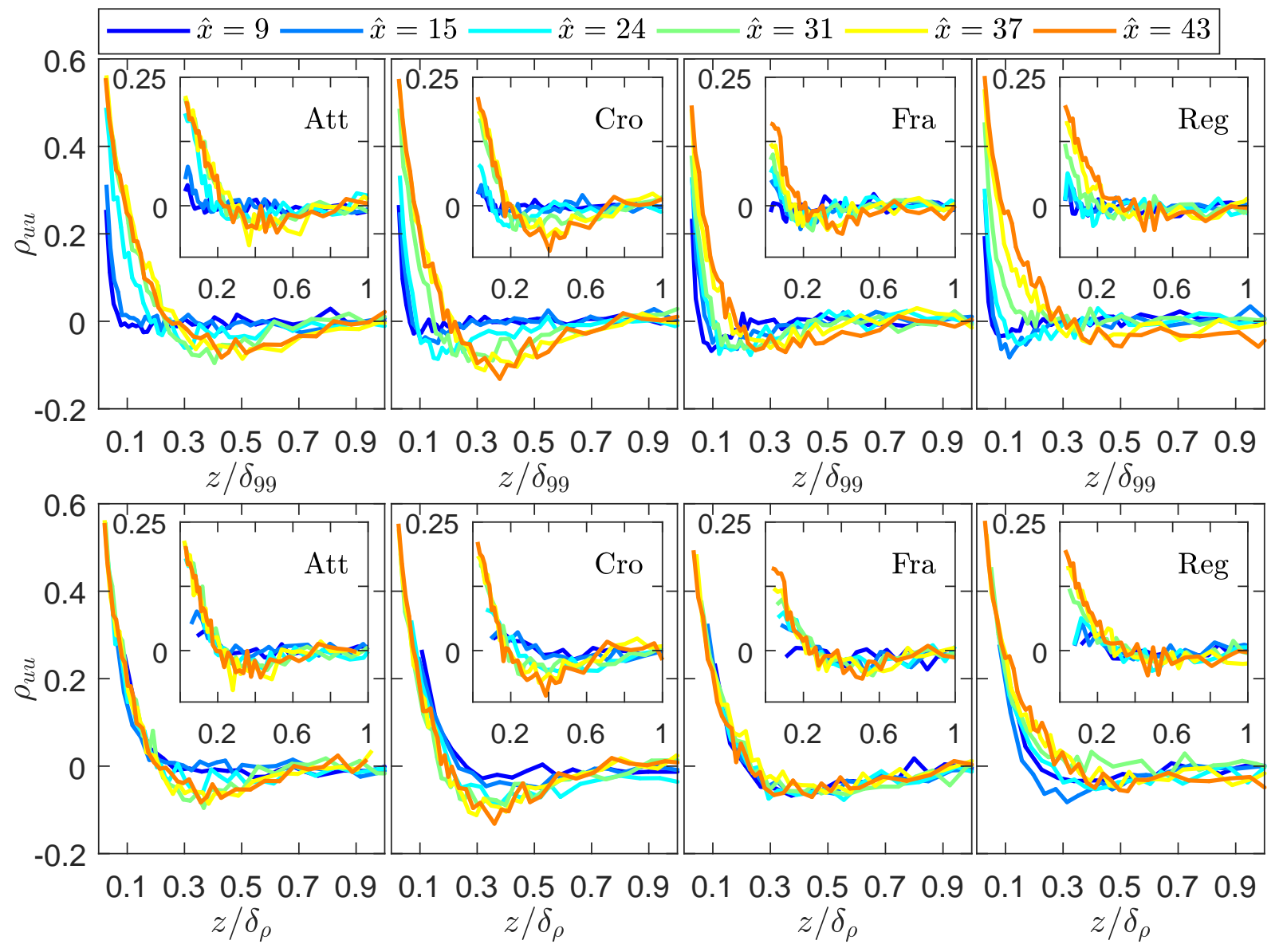

Figure 11: Profiles of the spanwise correlation of streamwise velocity $\rho_{u u}(z)$ at the beginning of the logarithmic region, $y+\approx 60, y / \delta_{99} \approx 0.02$, (main plots) and in the near-wall region, $y+\approx 4$ (insets), for various grids and streamwise locations normalized with $\delta_{99}$ (first row) and $\delta_{\rho}$ (second row). The grids are always located at $x_{g}=100 \mathrm{~mm}$ where the incoming boundary layer thickness $\delta^{I} \approx 0$.

Two-point spanwise measurements are taken using both WHW and FHW simultaneously. Two heights over 
structures are expected to appear. Correlation of the streamwise velocity in the spanwise direction is defined as

$$
\rho_{u u}(z)=\frac{\int_{0}^{\infty} u_{w h w}^{\prime}(z=0, t) u_{f h w}^{\prime}(z, t) \mathrm{d} t}{u_{w h w, r m s}^{\prime} u_{f h w, r m s}^{\prime}}
$$

where the minimum separation between the measurement points (given by the physical size of the hot wires) is approximately $4 \mathrm{~mm}$. A similar experimental arrangement was used in Rodríguez-López et al. (2016b).

The first row of figure 11 shows the spanwise correlation of streamwise velocity for several grids and streamwise locations normalized with $\delta_{99}$. The lack of collapse for different $\hat{x}$ is clearly observed, in particular, this effect is more pronounced closer to the grids. Note that, for $\hat{x} \leq 15, \rho_{u u}$ drops to 0 for $z / \delta_{99} \approx 0.05$, much smaller than the expected value of approximately 0.3 (Hutchins and Marusic, 2007a). Furthermore, for Att and Cro grids, very little negative correlation is found for $\hat{x}<24$. This contrasts with the natural TBL case, in which the turbulent structures of positive fluctuation are flanked by negative ones. It seems therefore reasonable to assume that the presence of the grids destroys this pattern. This effect is less clear for Fra and Reg grids suggesting that the wall behaviour is less disrupted in this case, which is in agreement with past observations regarding the interaction between outer and wall layers (c.f. sections 3.2 and 3.3). Both effects (rapid loss in correlation and lack of negative values) tend towards the expected values of a natural TBL with $x$, this is consistent with the growth of the internal layer which progressively tends to $\delta_{99}$. In particular, the typical arrangement of alternating spanwise structures is recovered for Att and Cro grids at $\hat{x} \approx 24$, this is the same straemwise location where the turbulence peak related with the shear layer is lost (c.f. figure 3). From this evidence, it could be conjectured that the disappearance of the shear-layer peak and the appearance of the large structures in the logarithmic layer are related. Moreover, the geometry of the grids (figure 2) also suggests that Att and Cro grids should destroy the turbulent structures in the wall region more effectively as a consequence of the larger concentration of bars close to $\hat{y}=0$.

Let us now normalize the spanwise separation with the new scaling thickness $\delta_{\rho}$ as shown in the second row of figure 11. The performance of $\delta_{e f f}$ as a scaling parameter is unsusprisingly worse, since $\delta_{\rho}$ is based in the two-point correlation hence it is more valid to describe $\rho_{u u}$ in other directions. Thus, for the sake of brevity only results scaled with $\delta_{\rho}$ are presented.

One can note that the collapse of Fra and Reg grids is significantly improved with respect to the degree of matching presented in $L_{11} / \delta_{\rho}$ (figure 10). The important result resides not only in the fact that the curves are shown to collapse amongst themselves, but also in the spanwise location of the minimum of the correlation, this is always located close to $z / \delta_{\rho} \approx 0.3$ as has been reported for natural TBLs (Ganapathisubramani et al., 2005; Hutchins and Marusic, 2007a; Rodríguez-López et al., 2016b). Hence, this provides consistent evidence for $\delta_{\rho}$ being the correct scaling for the wall layer forming under the grids' wake.

The spanwise organization present in the logarithmic layer shows its footprint in the near-wall region. This is shown in the insets of figure 11 , where the same correlations are plotted but this time at $y^{+} \approx 4$. The maximum correlation level is unsurprisingly smaller since the initial separation between the two hot wires is kept constant and typical eddy sizes are expected to be much smaller in this region than in the logarithmic layer (c.f. also 

${ }_{2} \delta_{\rho}$.

figure 10). However, a similar trend is observed for all the grids and streamwise locations which collapse with

Summarizing, large structures flanked by opposite sign fluctuations are destroyed in the vicinity of Att and Cro grids to form again after approximately 24 grid heights. For streamwise distances larger than this value and for the other two grids (at every $\hat{x}$ ) these large structures are detected in the logarithmic layer with a separation of approximately $0.3 \delta_{\rho}$ (analogous to a naturally developing TBL). As expected, it is shown that $\delta_{99}$ cannot be used as a scaling parameter, as it does not capture the physics of the wall layer. The footprint of these structures is also reflected in the very near-wall region with analogous separation $\left(\propto \delta_{\rho}\right)$. This constitutes clear evidence of the dominant scaling in the wall layer.

\subsection{Incoming boundary layer influence}

Having established the dependence of TBL properties on the thickness of the inner layer, $\delta_{\rho}$ or $\delta_{\text {eff }}$, a question arises regarding the potential influence of the incoming BL thickness on the flow downstream of the grids. In particular, note that the scaling based on $\delta_{\text {eff }}$ was severely challenged in the Cro case, where the grid's wake presented a larger interaction with the near-wall region. This influence was observed as a connection between the inner spectral peak and the shear layer region (c.f. figure 6). Moreover, it generated further difficulties for the determination of $\delta_{\text {eff }}$ from the $u_{r m s}^{\prime+}$ profile. It may be speculated that such scaling would exist even in this case but it cannot be detected with the present methodology. A similar effect (larger connection of the spectral contours between the inner and shear layers) can be obtained by the immersion of the grid in a progressively thicker TBL. This section therefore will study the spectra and integral length scale downstream of the various grids with different incoming TBLs.

The different incoming boundary layers (of thickness $\delta^{I}$ and momentum thickness $\theta^{I}$ ) are obtained by means of placing the grid at different streamwise locations where the TBL has grown to a larger thickness. All the measurements are taken at $\tilde{x}=8$, i.e. 8 grid heights downstream of the grid. Five different degrees of immersion are tested ranging from virtually 0 (the case studied up until now) to approximately $80 \%$ of the grid height. For the thickest TBL case, artificial tripping was used to deliberately overtrip the developing TBL. This trip was made of two rows of slender cylinders $20 \mathrm{~mm}$ high with their axis normal to the wall placed at $160 \mathrm{~mm}$ from the leading edge. Details of this experimental arrangement can be found in Rodríguez-López et al. (2016b,a) where it is also justified that the TBL properties at the considered streamwise location are sufficiently canonical and the trip influence seems to be forgotten (at least sufficiently for the present purpose). Two different thickness of the TBL can be considered, $\delta_{99}^{I}$ based on the mean velocity profile reaching $0.99 U_{\infty}$ or, alternatively, $\delta^{I}$ based on a fit of a composite profile to the outer layer (Chauhan et al., 2009; Rodríguez-López et al., 2015). Table 5 summarizes the main parameters of the various incoming TBLs.

Increasing the thickness of the incoming TBL implies that a larger fraction of the grid is subject to incoming turbulence (as opposed to the original case in which almost the whole flow impacting the grid was laminar). Furthermore, the length scale of the turbulence inside the incoming boundary layer will also increase (scaling 


\begin{tabular}{|c|c|c|c|c|c|c|c|}
\hline \multirow{5}{*}{1} & $\hat{x}_{g}$ & $\delta_{99}^{I}(\mathrm{~mm})$ & $\delta^{I}(\mathrm{~mm})$ & $\theta^{I}(\mathrm{~mm})$ & $R e_{\theta}$ & $u_{\tau}\left(\mathrm{ms}^{-1}\right)$ & $R e_{\tau}$ \\
\hline & 1 & 1.91 & 2.49 & 0.26 & 168 & 0.36 & 58 \\
\hline & 8 & 18.4 & 26.2 & 3.08 & 1891 & 0.40 & 687 \\
\hline & 19 & 33.2 & 44.7 & 4.22 & 2623 & 0.37 & 1066 \\
\hline & 36 & 44.6 & 58.2 & 5.81 & 3563 & 0.38 & 1419 \\
\hline$z$ & 36 & 59.9 & 78.6 & 8.53 & 5220 & 0.36 & 1805 \\
\hline
\end{tabular}

1: Estimation from Blasius laminar BL at $\hat{x}=1$.

2: With cylinders tripping based on Rodríguez-López et al. (2016b,a).

Table 5: Summary of TBL properties for various incoming TBL thickness. The friction velocity $u_{\tau}^{I}$ is extrapolated from the mean velocity profile (Rodríguez-López et al., 2015).

with its thickness, c.f. figure $12(\mathrm{~b}))$. This last effect is particularly important since it is anticipated that the 2 grids interact with incoming turbulence of similar lengths to their mesh size (e.g. Groth and Johansson, 1988).

3 Figure 12 shows the integral length scale for the various incoming boundary layers without the grid. Figure 12(b)

4 follows the expected trend for the natural TBL exhibiting a collapse for small $y$ followed by a plateau with a 5 slight Reynolds trend. This fact entails that $L_{11}$ grows with respect to the grid height (and consequently any 6 measurement of the mesh size) as shown in figure 12(a). One can observe that there is a clear increase in both 7 the fraction of grid influenced by the incoming TBL and also the typical length scale of this turbulence. Whilst 8 the near-wall region is strongly influenced by the presence of the wall, the vast majority of the grids are exposed 9 to turbulence of quasi constant $\hat{L}_{11}$.

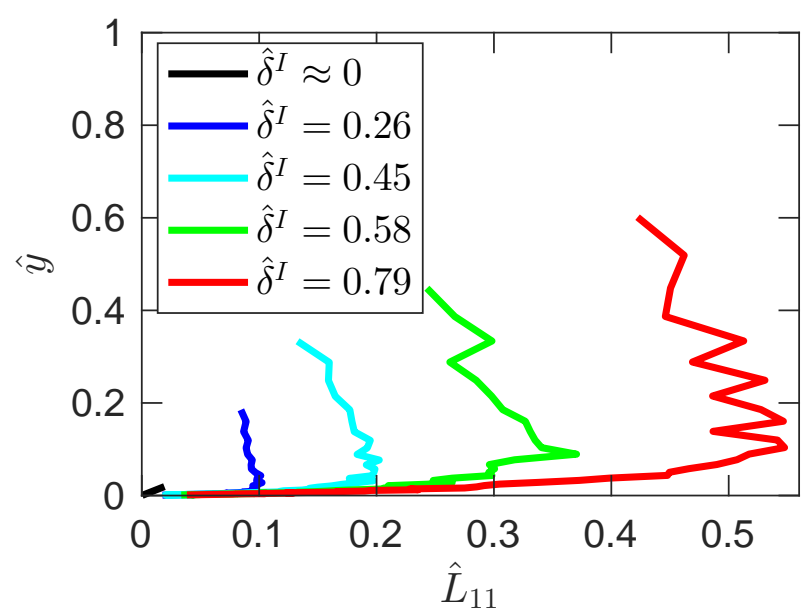

(a)

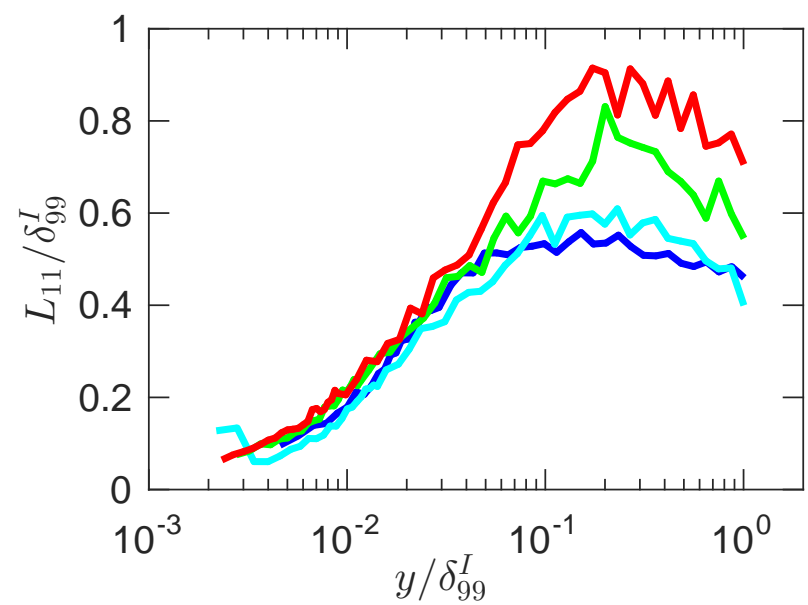

(b)

Figure 12: Wall-normal profile of integral length scale $L_{11}$, for different incoming boundary layers as a function of $y$ normalized with (a) the grid height $h$, and (b) the local TBL thickness $\delta_{99}^{I}$.

The increasing proportion of the grid influenced by the incoming turbulence can also be seen in the spectral contours. Figure 13 shows these contours for different incoming boundary layer thicknesses. The wall-normal extent of the region influenced by the near-wall turbulence is clearly seen to increase for larger incoming TBL. Furthermore, the dashed lines show the thickness of the incoming TBL ( $\delta^{I}$, based on mean-profile fitting) showing a clear correlation between the extent of the near-wall influenced region and the thickness of this layer. The behaviour of the other three grids is not shown for brevity but they show qualitatively similar trends.

The intensity of the peak related with the shear layer is seen to increase with $\delta^{I}$. This may be caused 
1 by two different reasons: firstly, increasing $\delta^{I}$ implies that the highly intermittent region of the TBL appears 2 at a similar height as the shear layer. This interaction may increase the fluctuations of the shear-layer peak. 3 Secondly, as in every naturally growing TBL, with increasing streamwise distance the TBL thickness, $\delta^{I}$, is 4 increased and the incoming shear velocity, $u_{\tau}^{I}$, is decreased. This implies that $u_{\tau}$ at $\tilde{x}=8$ is also smaller. Since 5 the outer peak is generated by the shear layer (not scaling in inner variables), this could be reflected in an 6 apparent increase of the peak's magnitude. On the other hand, profiles of $\hat{u}_{r m s}^{\prime}$ (nondimensionalized with $U_{\infty}$, not shown) display an increasing trend of this peak with $\delta^{I}$ which suggests the first hypothesis is more likely than the second one.

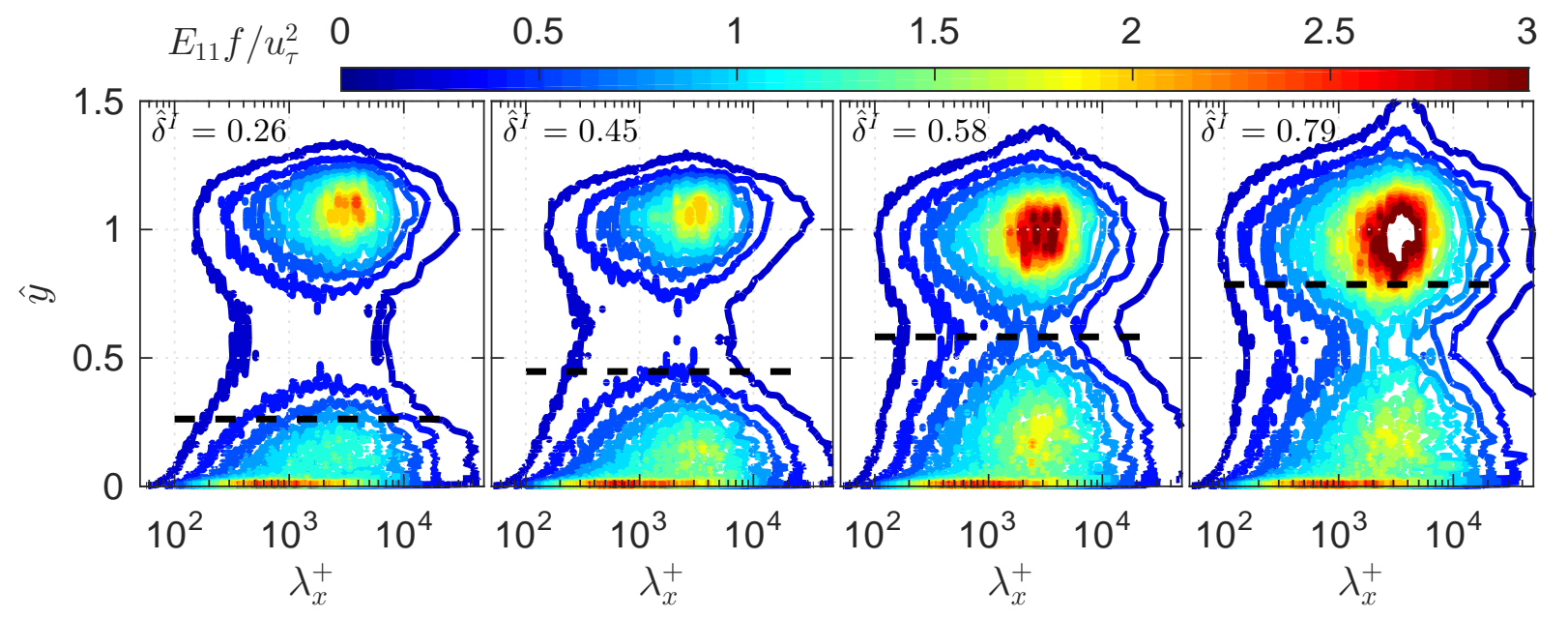

Figure 13: Inner-scaled premultiplied spectra, $E_{11}\left(u^{\prime}\right) f / u_{\tau}^{2}$, as a function of inner-scaled length scale, $\lambda_{x}^{+}$, and the wall-normal coordinate, $\hat{y}$, for various incoming boundary layers, $\delta^{I}$, (shown with the dashed lines, c.f. table 5). The grid is always $A t t$ and $\tilde{x}=8$.

The large turbulence intensity present in the shear layer has a further implication. As can be seen in figure 13, the penetration of turbulence that can be related with the near-wall behaviour seems to saturate for the largest $\delta^{I}$. In other words, despite the thickness of the incoming TBL is $\hat{\delta}^{I}=0.79$; the spectral contours seem to be narrowest at $\hat{y} \approx 0.6$. This might be due to the significantly stronger fluctuations of the shear layer which cover the near-wall behaviour. Contrastingly, the intensity of the shear-layer peak is significantly influenced by the presence of the thicker incoming TBL.

In practice, the largest prevalence of the near-wall region downstream of the grid may impose some differences on the scaling based on $\delta_{\rho}$ or $\delta_{\text {eff }}$ proposed above. The latter scaling was first suggested to represent the point at which the spectral contours are narrowest (i.e. the point which separates the wall and shear layer contributions). However, in this case, there seems to be a clear correlation between the thickness of the wall layer and the thickness of the incoming TBL. A possible explanation could be that the wall layer (with equivalent thickness $\delta_{\text {eff }}$ or $\delta_{\rho}$ ) is closely determined by the thickness of the incoming TBL close to the grid and it progressively grows with $\hat{x}$ until reaching $\delta_{99}$. However, note that this would suggest that a larger $\delta^{I}$ implies a shorter distance for $\delta_{\text {eff }}$ to become $\delta_{99}$, which seems unlikely due to the stronger intensity of the shear-layer peak. Thus it seems that growth rates of $\delta_{\text {eff }}$ may change for different $\delta^{I}$. However, due to the limited size of the wind tunnel this hypothesis could not be tested and remains an open question. 

${ }_{4} \delta_{e f f} \sim \delta_{\rho} \sim \delta^{I}$. Thus as a first approach $\delta_{\text {eff }}=\delta_{99}^{I}$, note that this imposes a clear problem at $\delta^{I} \approx 0$ where ${ }_{5} \quad \delta_{\text {eff }} \neq 0$. However, this thickness is known from section 3.3 .

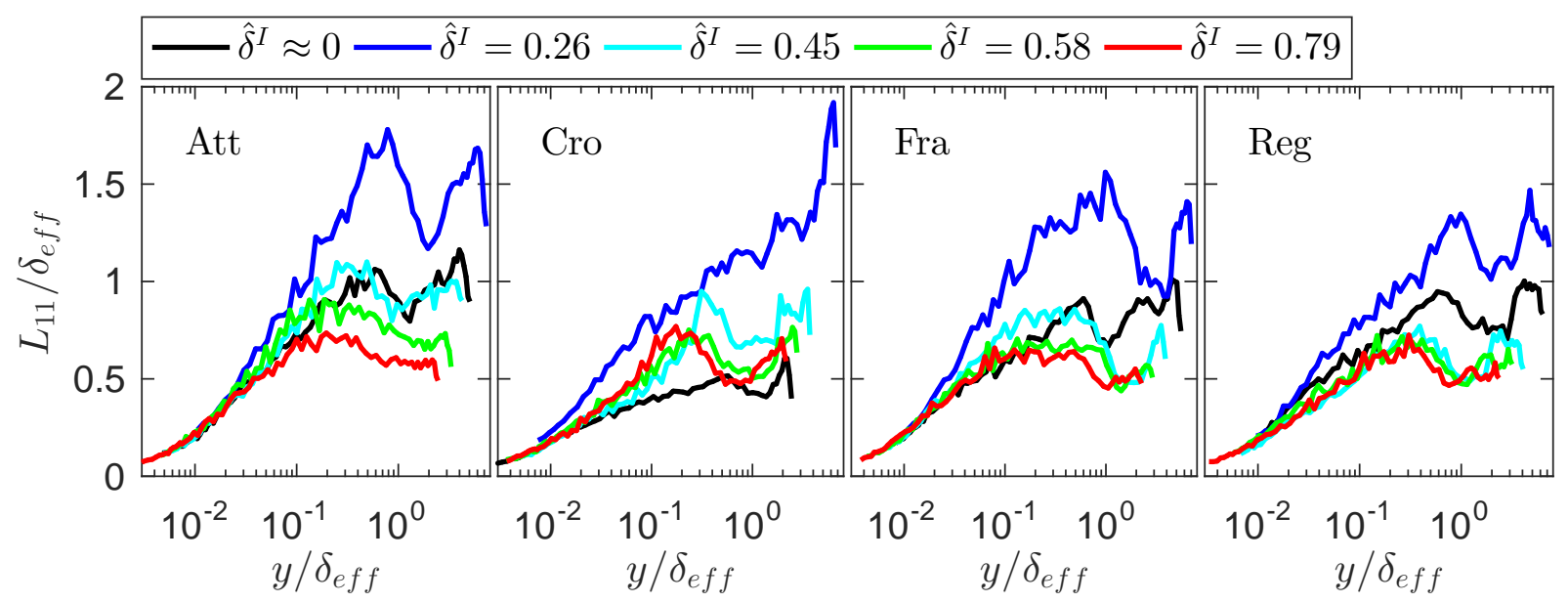

Figure 14: Profiles of integral length scale $L_{11}$, for various grids and different incoming boundary layers normalized with $\delta_{\text {eff }}=\delta_{99}^{I}$ except for the case $\delta^{I} \approx 0$ where $\delta_{\text {eff }}$ is calculated as described in section 3.3. The

streamwise location is always $\tilde{x}=8$.

What can be easily tested with the current dataset is the scaling of wall-normal profiles of longitudinal integral scale. It has been proposed that the $\delta_{99}$ scaling fails to capture the physics in the wall layer which scale with its local thickness $\delta_{\text {eff }}$. For increasing incoming TBL thickness it has also been hypothesized that

Figure 14 shows the proposed scaling for different grids and incoming TBLs. Despite the collapse of the curves being slightly poorer than for other aforementioned examples, it seems plausible to conclude that the longitudinal integral length scale, $L_{11}$, displays a satisfactory scaling with $\delta_{e f f}=\delta_{99}^{I}$. The fact that the $\hat{\delta}^{I}=0.26$ case shows a poorer scaling than the other cases may be explained by the same logic as for the $\delta^{I} \approx 0$ : in the streamwise fetch between the grid and the measurement location $(\tilde{x}=8)$ the inner layer is growing. As previously discussed, this growth rate can be assumed to be dependent on the initial thickness of the TBL (slower growth for larger $\left.\delta^{I}\right)$. This implies that, for small incoming boundary layers $\left(\hat{\delta}^{I} \lesssim 0.2\right)$, the efective thickness, $\delta_{e f f}$, would be smaller than $\delta_{99}^{I}$ at the measurement station $\tilde{x}=8$. Contrastingly, $\delta_{e f f}=\delta_{99}^{I}$ is a much better approach for large incoming boundary layers $\hat{\delta}^{I} \gtrsim 0.4$. In fact, note that the collapse for the three largest incoming TBLs is strikingly better than for any other case, supporting this hypothesis.

Summarizing, the appearance of two well-distinguished layers (dominated by near-wall and shear layer turbulence respectively) has been shown for different degrees of immersion of the grid in a TBL. It seems that, for a fixed streamwise distance downstream of the grid, the thickness of the inner layer is set by the thickness of the incoming TBL. However, different growth rates of this inner layer are hypothesized for different $\delta^{I}$. This is also confirmed by measurements at low immersions in which $\delta_{\text {eff }}=\delta_{99}^{I}$ does not seem to hold, probably because $\delta_{\text {eff }}$ has grown faster from the initially small $\delta_{99}^{I}$. 


\section{Conclusions}

A comprehensive flow characterization using one- and two-point hot-wire anemometry has been conducted downstream of single- and multi-scale wall-mounted highly porous fences. The bulk behaviour of the grids, studied by means of their drag, seems to be mainly dependent on their blockage ratio (which is kept the same for the various grids). However, slightly smaller value is detected for the Cro grid most likely due to the appearance of non-zero vertical velocity and the smaller aspect ratio of its bars. From another perspective, turbulent properties are shown to be dependent on the different distributions of blockage formed of bars of distinct sizes and orientations. These grids therefore generate a highly complex and inhomogeneous flow in their vicinity which interacts with the wall and evolves progressively towards a canonical turbulent boundary layer state. In this adaptation region, the turbulence of the bars' wakes seems to decay much faster than that generated by either the wall or the shear layer appearing between the grid's wake and the freestream. This is most likely due to the large mean shear appearing in these two regions which promotes turbulence production enhancing the fluctuations at these layers. Consequently the flow physics in the adaptation region are dominated by two different mechanisms depending on whether one considers the wall layer or the shear layer. In particular, spectra at large distances from the wall are shown to be clearly related with shear-layer thickness and, similarly, spectra in the near-wall region seem to follow the typical scaling of wall-bounded flows $\left(\lambda_{x}^{+} \approx 1000\right)$.

The appearance of this clear separation of spectral behaviour along the wall-normal coordinate has enabled the definition a well-differentiated wall layer of effective thickness $\delta_{e f f}$. The thickness of this layer can also be established as a function of the point at which the wall normal profile of streamwise velocity correlation falls below a certain threshold $\left(\delta_{\rho}\right)$. Both scalings can provide a measurement of the thickness of the wall layer although the latter seems to provide a more accurate description. The thickness of the wall layer is defined as the thickness of a naturally growing TBL which presents the same properties of that inner layer which develops under the influence of the fences' wakes. Both $\delta_{\rho}$ and $\delta_{\text {eff }}$ are shown to increase with $x$ approaching $\delta_{99}$ or even exceeding it.

Since this effective thickness can be understood as the upper boundary condition of the wall layer; $\delta_{\rho}$ dominates the scaling of velocity fluctuations inside this region. In particular, a satisfactory scaling in terms of $\delta_{\rho}$ is shown to hold for root-mean-square level of the wall shear stress fluctuations $\left(\tau_{w, r m s}^{\prime+}\right)$, longitudinal integral length scale $\left(L_{11}\right)$ and spanwise turbulent structure $\left(\rho_{u u}(z)\right)$. Furthermore, details of the spanwise turbulent structure has also shown that large structures flanked by opposite sign fluctuations are not present for Att and Cro grids before $24 h$. For $\hat{x}>24$ and for the other two grids (every $x$ ) these large structures appear in the logarithmic layer with a separation of $0.3 \delta_{\rho}$ (similar to a natural TBL).

For cases where the incoming boundary layer was finite and comparable to the grid height $h$; the effective thickness of this inner layer is shown to scale with the thickness of the incoming TBL, $\delta^{I}$; at least for values of $\hat{\delta}^{I} \gtrsim 0.4$. Furthermore, it is conjectured that the growth rate of $\delta_{\text {eff }}$ is also dependent on the incoming TBL thickness. In particular, it seems to grow faster for smaller $\delta^{I}$.

Despite the length of the adaptation region and the potential recovery of canonical TBL properties down- 
1

2 3

\section{${ }_{4}$ A Appendix: WHW calibration} of the spanwise structure.

stream of it reamain an open question; it seems reasonable to hypothesize that Fra or Reg grids would perform better as TBL trips because of its smaller interaction between wall and wake layers and the lack of destruction

Calibration of wall-mounted hot wires (WHW) presents a number of significant challenges that should be carefully considered. Firstly, these devices may only be traversed over a limited spatial extent, which does not include the freestream. Secondly, in most of the cases their fragility makes it impossible to remove them from their position in order to use a commercial calibrator or place them in the freestream. Moreover, the relative orientation of the prong-wire ensemble with respect to the incoming flow should be the same for calibration and experiment. If accurate values of the calibration are not required, one can estimate the hot wire response by calibrating the WHW against another hot wire immersed in the TBL shear flow (see Rodríguez-López et al., 2016b). However, in order to obtain the true values of the velocity an alternative procedure must be followed.

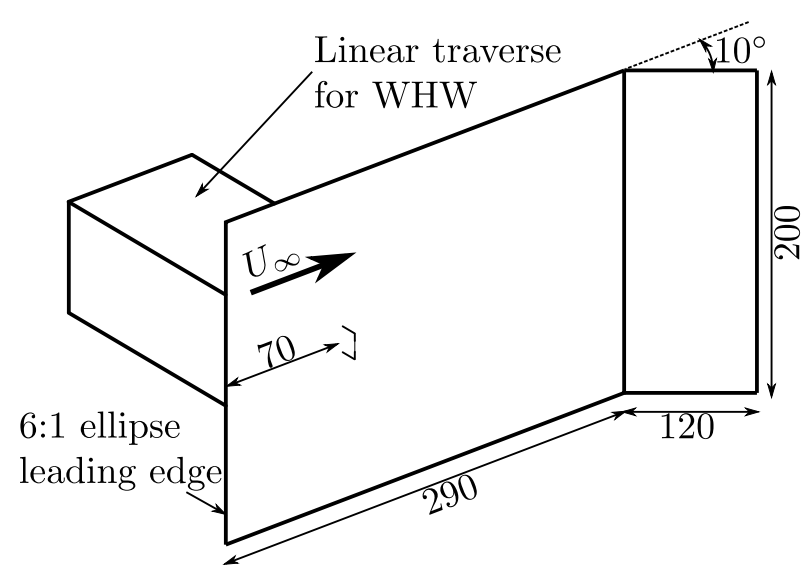

(a)

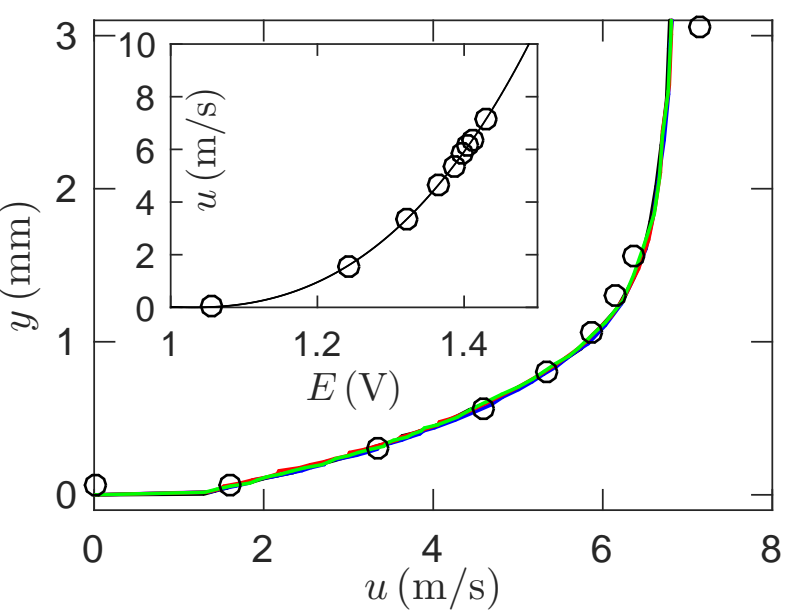

(b)

Figure 15: (a) Sketch of the WHW calibrator device (Not to scale), flow goes left to right. Dimensions are in millimetres. (b) Solid lines show the velocity profiles at the WHW location (measured with another hot wire). Empty circles show the points acquired by the WHW. Inset shows the King's law (solid black line) fit to those points.

The present methodology consists of the manufacturing of a small calibration plate such that the boundary layer developing on its surface remains laminar and smaller than the largest height that the WHW can reach over the wall $\left(y_{\max }^{W H W} \approx 3 \mathrm{~mm}\right)$. Furthermore, the WHW will always be located in the near-wall region, therefore the calibration should focus more on low velocity values rather than high speed. A small flat plate is made from a $4 \mathrm{~mm}$ thick aluminium sheet provided with a 3d-printed elliptic leading edge and a flap to compensate for the blockage of the WHW's linear traverse on the back of the plate. A sketch of the arrangement is shown in figure 15(a). In order to know the velocity at the WHW location, several profiles were acquired with another hot wire to ensure repeatability of the measurements. Figure 15(b) shows these velocity profiles where one can appreciate the similarity between the various acquisitions. After this velocity profile is reliably 
known, the calibration procedure consisted of sampling the WHW for $20 \mathrm{~s}$ at different wall-normal locations (from its minimum position $y_{0}^{W H W}=0.18 \mathrm{~mm}$ until $1.5 \mathrm{~mm}$ in steps of $0.25 \mathrm{~mm}$ and a further measurement at $\left.y^{W H W}=3 \mathrm{~mm}\right)$. The WHW response at these locations is associated with the known velocity at the same $y$ which is known from the velocity profiles acquisition. Once the response of the WHW is known, the coefficients $A, B$ and $C$ of the King's law $\left(E^{2}=A+B U^{C}\right)$ are estimated by a least-squares fit.

Figure 15(b) shows the values of the WHW response compared with the velocity profile measured before the calibration procedure. The agreement between these measurements and the velocity profiles is remarkably good at low velocities. However, it diverges slightly for the largest height, probably as a consequence of the interference of the prongs with the incoming fluid. Note that, at this position, the prong-to-prong distance is similar to its elevation over the wall hence some interaction may be expected. This is not expected to affect the results since this interference primarily affects the mean value and the current study only uses the fluctuations. The agreement with the King's law is shown in the inset of figure 15(b) also displaying a remarkable agreement.

\section{B Appendix: $C_{d}$ determination}

Let us consider a two-dimensional domain with its lower limit in the wall and the upper limit taken to be an undisrupted streamline far enough from the wall and the grid such that no mass or momentum cross this boundary. The subscripts 1 and 2 will denote the inlet (left) and outlet (right) of this domain respectively. We can write the mean integral momentum balance for this domain as:

$$
\int_{0}^{y_{1}} \rho \bar{u}_{1}^{2}(y) \mathrm{d} y+\int_{0}^{y_{1}} \bar{p}_{1}(y) \mathrm{d} y-\int_{0}^{y_{2}} \rho \bar{u}_{2}^{2}(y) \mathrm{d} y-\int_{0}^{y_{2}} \bar{p}_{2}(y) \mathrm{d} y=D+\int_{x_{1}}^{x_{2}} \bar{\tau}_{w}(x) \mathrm{d} x .
$$

The selection of the region of the flow is not trivial and one should take some considerations prior to its determination. On the one hand taking $x_{2}$ close to the grid (e.g. Dong et al., 2008) enables us to neglect the contribution of the shear stress term, which usually is difficult to measure or estimate. On the other hand, close to the grid the pressure contribution may be important and the spanwise inhomogeneities of the grid would make it impossible to assume two-dimensionality of the measurement domain. Seginer (1972) showed that the contribution of the term in $p_{2}$ reduces drastically for $\hat{x}_{2}-\hat{x}_{1} \approx 10$. Furthermore, the simplification of the 3D problem into a $2 \mathrm{D}$ domain can only be done after the spanwise inhomogeneities have dissappeared. Preliminary measurements (not shown for brevity) confirm that the spanwise homogeneity of the mean and rms profiles are significantly reduced for $\tilde{x}>5$. The downstream limit of the domain should be therefore located further from that location. In order to find a compromise, the selected domain will have $x_{1}$ upstream of the grid and $x_{2}=8 h$ downstream of it. Moreover, since the upper edge of the domain is taken to be sufficiently far from the wall, we can assume that $y_{2} \approx y_{1}$ for simplicity although it does not have any effect on the results.

With respect to the upstream limit of the domain, this will be taken to be $\tilde{x}_{1}=-1$, that is, at the leading edge of the flat plate. At this point the momentum thickness $\theta_{1}=0$ independently of the grid being mounted or not. 
For long streamwise domains (as the one considered), Seginer (1972) reported a low contribution of the pressure terms to the drag balance. Furthermore, under boundary layer assumptions, we could assume $\bar{p}(y) \approx \bar{p}_{w}$ independent of $y$. Note in figure 1 (a) that for $\tilde{x}=8 \leftrightarrow x=0.9 \mathrm{~m}$, the wall pressure for the various grid cases is approximately the same as in the natural case. Therefore, we can assume that the flow is quasi-zero pressure gradient since variations in $C_{p}$ are always smaller than $2 \%$. Note that, strictly speaking $\bar{p}(y) \approx \bar{p}_{w}$ may ot hold in the grids' wake. However, preliminary measurements (not shown) reported a decrease of the wall-normal velocity for streamwise locations $\tilde{x}>2$ which may support the use of this approximation. Hence it seems reasonable to neglect the contribution to the drag of the pressure difference across the domain.

Under these assumptions, non-dimensionalizing the equations with the grid height $h$, and the freestream velocity $U_{\infty}$, and expressing the velocity profiles in terms of the momentum thickness $\theta=\int_{0}^{\infty}[\hat{u}(1-\hat{u})] \mathrm{d} y$; equation (5) results:

$$
C_{d} \approx \frac{2}{h}\left[\theta_{2}-\frac{1}{\rho U_{\infty}^{2}} \int_{x_{1}}^{x_{2}} \bar{\tau}_{w}(x) \mathrm{d} x\right]
$$

Where the wall shear stress term remains unknown because measurements of $\bar{\tau}_{w}$ are not available for every $x_{1} \leq x \leq x_{2}$. However, $\bar{\tau}_{w}$ is known at station 2 (Rodríguez-López et al., 2017, and Table 2). In order to provide a meaningful estimation for the last term, let us consider the natural TBL case (without any grid installed), for this case we can write $\int_{x_{1}}^{x_{2}} \bar{\tau}_{w}^{N} \mathrm{~d} x \propto \theta_{2}^{N}$, where superscript $N$ denotes the natural case and, as mentioned above, $\theta_{1}^{N}=\theta_{1}=0$. Given that we have the measurement of the wall shear stress at $x_{2}$, we can use this information to approximate the last term in equation (6) as:

$$
\frac{1}{\rho U_{\infty}^{2}} \int_{x_{1}}^{x_{2}} \bar{\tau}_{w}(x) \mathrm{d} x \approx \tau^{G N} \frac{1}{\rho U_{\infty}^{2}} \int_{x_{1}}^{x_{2}} \bar{\tau}_{w}^{N}(x) \mathrm{d} x \approx \tau^{G N} \theta_{2}^{N}
$$

where $\tau^{G N}=\bar{\tau}_{w}\left(x_{2}\right) / \bar{\tau}_{w}^{N}\left(x_{2}\right)$ is the relationship between the wall shear stress in the grid and the natural cases at $x_{2}$. Note that the history of the wall shear stress between $x_{1}$ and $x_{2}$ is not known but we know the streamwise development of $\bar{\tau}_{w}(x)$ for $x>x_{2}$ as shown in Table 2 . There, one can see that the shear stress remains relatively constant along $x>x_{2}$ which sustains the use of $\bar{\tau}_{w}\left(x_{2}\right)$ as a control parameter for $\bar{\tau}_{w}\left(x<x_{2}\right)$. In any case, it has been shown (Table 3) that the contribution of the wall shear stress term to the drag determination is smaller than $10 \%$ hence variations in the modelization of this term have a rather limited impact in $C_{d}$.

Thus the drag coefficient of the grids can be estimated as

$$
C_{d} \approx \frac{2}{h}\left[\theta_{2}-\theta_{2}^{N} \tau^{G N}\right]
$$

\section{References}

Alfredsson, P. H., Segalini, A., and Örlü, R. (2011). A new scaling for the streamwise turbulence intensity in wall-bounded turbulent flows and what it tells us about the "outer" peak. Physics of Fluids, 23(4):041702.

Andreopoulos, J. and Wood, D. H. (1982). The response of a turbulent boundary layer to a short length of surface roughness. Journal of Fluid Mechanics, 118:143-164. 
Antonia, R. A. and Luxton, R. E. (1971). The response of a turbulent boundary layer to a step change in surface roughness Part 1 . Smooth to rough. Journal of Fluid Mechanics, 48(4):721-761.

Boppe, R. S., Neu, W. L., and Shuai, H. (1999). Large-scale motions in the marine atmospheric surface layer. Boundary-Layer Meteorology, 92(2):165-183.

Brown, G. L. and Thomas, A. S. W. (1977). Large structure in a turbulent boundary layer. Physics of Fluids, 20(10):243-252.

Brunn, H. (1995). Hot-wire anemometry. UOP Oxford, Oxford, United Kingdom.

Castro, I. (1971). Wake characteristics of two-dimensional perforated plates normal to an air-stream. Journal of Fluid Mechanics, 46(3):599-609.

Chauhan, K. A., Monkewitz, P. A., and Nagib, H. M. (2009). Criteria for assessing experiments in zero pressure gradient boundary layers. Fluid Dynamics Research, 41(2):021404.

Chernyshenko, S. I. and Baig, M. F. (2005a). Streaks and vortices in near-wall turbulence. Philosophical Transactions of the Royal Society, 363:1097-107.

Chernyshenko, S. I. and Baig, M. F. (2005b). The mechanism of streak formation in near-wall turbulence. Journal of Fluid Mechanics, 544:99-131.

Chew, Y. T., Khoo, B. C., Lim, C. P., and Teo, C. J. (1998). Dynamic response of hot-wire-anemometer. PartII: A flush-mounted hot-wire and hot-film probes for wall shear stress measurements. Measurement Science $\mathcal{E}$ Technology, 9(5):764-778.

Counihan, J. (1969). An improved method of simulating an atmospheric boundary layer in a wind tunnel. Atmospheric Environment, 3(2):197-214.

Dairay, T., Obligado, M., and Vassilicos, J. C. (2015). Non-equilibrium scaling laws in axisymmetric turbulent wakes. Journal of Fluid Mechanics, 781:166-195.

Degraa, D. B., Webster, D. R., and Eaton, J. K. (1999). The effect of Reynolds number on boundary layer turbulence. Experimental Thermal and Fluid Science, 18:341-346.

Del Alamo, J. C. and Jimenez, J. (2009). Estimation of turbulent convection velocities and corrections to Taylor's approximation. Journal of Fluid Mechanics, 640:5-26.

Díaz-Daniel, C., Laizet, S., and Vassilicos, J. C. (2017). Wall shear stress fluctuations: mixed scaling and their effects on velocity fluctuations in a turbulent boundary layer. Physical Review Fluids, Under review.

Dogan, E., Hanson, R. E., and Ganapathisubramani, B. (2016). Interactions of large-scale free-stream turbulence with turbulent boundary layers. Journal of Fluid Mechanics, 802:79-107.

Dong, Z., Mu, Q., Luo, W., Qinan, G., Lu, P., and Wang, H. (2008). An analysis of drag force and moment for upright porous wind fences. Journal of Geophysical Research, 113(D4):1-8.

Efros, V. and Krogstad, P.-A. (2011). Development of a turbulent boundary layer after a step from smooth to rough surface. Experiments in Fluids, 51(6):1563-1575.

Erm, L. P. and Joubert, P. N. (1991). Low-Reynolds-number turbulent boundary layers. Journal of Fluid Mechanics, 230:1-44.

Ganapathisubramani, B., Hutchins, N., Hambleton, W. T., Longmire, E. K., and Marusic, I. (2005). Investi- 
gation of large-scale coherence in a turbulent boundary layer using two-point correlations. Journal of Fluid Mechanics, 524(2005):57-80.

Geng, C., He, G., Wang, Y., Xu, C., Lozano-Durán, A., and Wallace, J. M. (2015). Taylor's hypothesis in turbulent channel flow considered using a transport equation analysis. Physics of Fluids, 27(2):025111.

Goh, K., Geipel, P., and Lindstedt, R. (2014). Lean premixed opposed jet flames in fractal grid generated multiscale turbulence. Combustion and Flame, 161(9):2419-2434.

Goh, K. H. H., Geipel, P., Hampp, F., and Lindstedt, R. P. (2013). Flames in fractal grid generated turbulence. Fluid Dynamics Research, 45(6):061403.

Gomes-Fernandes, R., Ganapathisubramani, B., and Vassilicos, J. C. (2012). Particle image velocimetry study of fractal-generated turbulence. Journal of Fluid Mechanics, 711:306-336.

Good, M. and Joubert, P. N. (1968). The form drag of two-dimensional bluff-plates immersed in turbulent boundary layers. Journal of Fluid Mechanics, 31(3):547-582.

Groth, J. and Johansson, A. V. (1988). Turbulence reduction by screens. Journal of Fluid Mechanics, 197:139155.

Guan, D., Zhang, Y., and Zhu, T. (2003). A wind-tunnel study of windbreak drag. Agricultural and Forest Meteorology, 118(1-2):75-84.

Hanson, R. E. and Ganapathisubramani, B. (2016). Development of turbulent boundary layers past a step change in wall roughness. Journal of Fluid Mechanics, 795:494-523.

Hoerner, S. F. (1965). Fluid-dynamic drag: practical information on aerodynamic drag and hydrodynamic resistance. Hoerner Fluid Dynamics, 2nd edition.

Hultmark, M., Ashok, A., and Smits, A. J. (2011). A new criterion for end-conduction effects in hot-wire anemometry. Measurement Science and Technology, 22(5):055401.

Hunt, J. C. R. and Fernholz, H. (1975). Wind-tunnel simulation of the atmospheric boundary layer: a report on Euromech 50. Journal of Fluid Mechanics, 70(3):543-559.

Hurst, D. and Vassilicos, J. C. (2007). Scalings and decay of fractal-generated turbulence. Physics of Fluids, 19(3):035103.

Hutchins, N. and Marusic, I. (2007a). Evidence of very long meandering features in the logarithmic region of turbulent boundary layers. Journal of Fluid Mechanics, 579:1-28.

Hutchins, N. and Marusic, I. (2007b). Large-scale influences in near-wall turbulence. Philosophical transactions. Series A, Mathematical, physical, and engineering sciences, 365(1852):647-64.

Hutchins, N., Nickels, T. B., Marusic, I., and Chong, M. S. (2009). Hot-wire spatial resolution issues in wallbounded turbulence. Journal of Fluid Mechanics, 635:103-136.

Jimenez, J. (2004). Turbulent Flows Over Rough Walls. Annual Review of Fluid Mechanics, 36(1):173-196.

Keylock, C. J., Nishimura, K., Nemoto, M., and Ito, Y. (2012). The flow structure in the wake of a fractal fence and the absence of an inertial regime. Environmental Fluid Mechanics, 12(3):227-250.

Li, B. and Sherman, D. J. (2015). Aerodynamics and morphodynamics of sand fences: A review. Aeolian Research, 17:33-48. 
Marusic, I. and Heuer, W. D. C. (2007). Reynolds number invariance of the structure inclination angle in wall turbulence. Physical Review Letters, 99(11):114504.

Marusic, I. and Kunkel, G. J. (2003). Streamwise turbulence intensity formulation for flat-plate boundary layers. Physics of Fluids, 15(8):2461-2464.

Marusic, I., McKeon, B. J., Monkewitz, P. A., Nagib, H. M., Smits, A. J., and Sreenivasan, K. R. (2010). Wall-bounded turbulent flows at high Reynolds numbers: Recent advances and key issues. Physics of Fluids, $22(6): 065103$.

Mathis, R., Hutchins, N., and Marusic, I. (2011). A predictive inner-outer model for streamwise turbulence statistics in wall-bounded flows. Journal of Fluid Mechanics, 681:537-566.

Mathis, R., Marusic, I., Chernyshenko, S. I., and Hutchins, N. (2013). Estimating wall-shear-stress fluctuations given an outer region input. Journal of Fluid Mechanics, 715:163-180.

Mazellier, N. and Vassilicos, J. C. (2010). Turbulence without Richardson-Kolmogorov cascade. Phys. Fluids, 22(7):075101.

Melina, G., Bruce, P. J. K., and Vassilicos, J. C. (2016). Vortex shedding effects in grid-generated turbulence. Physical Review Fluids, 1(4):044402.

Miller, M., Estejab, B., and Bailey, S. C. C. (2014). Evaluation of hot-wire spatial filtering corrections for wall turbulence and correction for end-conduction effects. Experiments in Fluids, 55(5):1735.

Nedić, J., Ganapathisubramani, B., Vassilicos, J. C., Borée, J., Brizzi, L. E., and Spohn, A. (2012). Aeroacoustic Performance of Fractal Spoilers. AIAA Journal, 50(12):2695-2710.

Nedić, J. and Vassilicos, J. C. (2015). Vortex shedding and aerodynamic performance of an airfoil with multiscale trailing edge modifications. AIAA Journal, 53(11):1-24.

Örlü, R. and Schlatter, P. (2013). Comparison of experiments and simulations for zero pressure gradient turbulent boundary layers at moderate Reynolds numbers. Experiments in Fluids, 54(6):1547.

Ranga-Raju, K. G., Garde, R. J., Singh, S. K., and Sing, N. (1988). Experimental study on characteristics of flow past porous fences. Journal of Wind Engineering and Industrial Aerodynamics, 29(1-3):155-163.

Robinson, S. K. (1986). Instantaneous velocity profile measurements in a turbulent boundary layer. Chemical Engineering Communications, 43(4):347-369.

Rodríguez-López, E., Bruce, P. J. K., and Buxton, O. R. H. (2015). A robust post-processing method to determine skin friction in turbulent boundary layers from the velocity profile. Experiments in Fluids, $56(4): 68$.

Rodríguez-López, E., Bruce, P. J. K., and Buxton, O. R. H. (2016a). Near field development of artificially generated high Reynolds number turbulent boundary layers. Physical Review Fluids, 1(7):074401.

Rodríguez-López, E., Bruce, P. J. K., and Buxton, O. R. H. (2016b). On the formation mechanisms of artificially generated high reynolds number turbulent boundary layers. Boundary-Layer Meteorology, 160(2):201-224.

Rodríguez-López, E., Bruce, P. J. K., and Buxton, O. R. H. (2017). Experimental measurement of wall shear stress in strongly disrupted flows. Journal of Turbulence, 18(3):271-290.

Schlatter, P. and Örlü, R. (2010). Assessment of direct numerical simulation data of turbulent boundary layers. Journal of Fluid Mechanics, 659:116-126. 
1 Schlatter, P. and Örlü, R. (2012). Turbulent boundary layers at moderate Reynolds numbers: inflow length 2 and tripping effects. Journal of Fluid Mechanics, 710:5-34.

3 Seginer, I. (1972). Windbreak drag calculated from the horizontal velocity field. Boundary-Layer Meteorology, $4 \quad 3(1): 87-97$.

5 Sillero, J. A., Jimenez, J., and Moser, R. D. (2013). One-point statistics for turbulent wall-bounded flows at $6 \quad$ Reynolds numbers up to $\delta\{\backslash$ approx\}2000. Physics of Fluids, 25(10):105102.

7 Steiros, K., Bruce, P. J. K., Buxton, O. R. H., and Vassilicos, J. C. (2017). Power consumption and form drag 8 of regular and fractal-shaped turbines in a stirred tank. AIChE Journal, 63(2):843-854.

9 Taylor, G. (1963). The Scientific Papers of G. I. Taylor. Vol. III. Aerodynamics and the Mechanics of Projectiles and Explosions. Cambridge University Press, Cambridge. Townsend, A. (1976). The Structure of Turbulent Shear Flow. Cambridge university Press, Cambridge and New York, 2 edition. Valente, P. C. and Vassilicos, J. C. (2014). The non-equilibrium region of grid-generated decaying turbulence. Journal of Fluid Mechanics, 744:8-37.

15 Wilson, J. D. (1987). On the choice of a windbreak porosity profile. Boundary-Layer Meteorology, 38(1):37-49. Woodruff, N. P., Fryrear, D. W., and Lyles, L. (1963). Engineering similitude and momentum transfer principles applied to shelterbelt studies. Transactions of the ASME, 6(1):41-47. 